Review Article

\title{
Pipeline Performances under Earthquake-Induced Soil Liquefaction: State of the Art on Real Observations, Model Tests, and Numerical Simulations
}

\author{
Massimina Castiglia, Tony Fierro, and Filippo Santucci de Magistris \\ DiBT Department, University of Molise, Campobasso, Italy \\ Correspondence should be addressed to Filippo Santucci de Magistris; filippo.santucci@unimol.it
}

Received 19 March 2020; Revised 2 July 2020; Accepted 20 July 2020; Published 3 August 2020

Academic Editor: Jie Zhang

Copyright (C) 2020 Massimina Castiglia et al. This is an open access article distributed under the Creative Commons Attribution License, which permits unrestricted use, distribution, and reproduction in any medium, provided the original work is properly cited.

\begin{abstract}
The design and the manufacture of the oil and gas pipelines are being improved over the years in response to the observed damages and related disastrous effects. The improvements are possible, thanks to the increasing knowledge about pipeline performances in specific contexts. The seismic hazard on buried pipelines has always been of major concern, and the earthquake-induced soil liquefaction effects are among the most important issues to be accounted for in the design. Experiences based on case histories, experimental modelling, and numerical simulations represent the source of understanding of the involved mechanisms, the affecting parameters, and the structure response. Recently, all these aspects are becoming more accurate, thanks to the use of monitoring systems. The protection of pipelines from the seismic hazard is a crucial and challenging issue. This paper provides an overview of the research that has been conducted over the years in the specific framework of soil liquefaction phenomenon. Case histories on pipeline performances, commonly adopted analytical methods, and results of model tests and numerical simulations are summarized with main focus on the level of knowledge achieved up to date and the existing limitations that represent open issues for further development of the research. This study represents a useful background to be adopted from academics and practitioners in order to enhance the methods of analyses of the pipelines, thus improving their performances in the applications of the oil and gas industry.
\end{abstract}

\section{Introduction}

Different scenarios need to be considered for pipeline design due to its deployment in large areas. If these structures cross regions of high seismicity, multiple serious problems might arise for these long-track buried structures and, among these, the soil liquefaction is of major interest. Several permanent ground movements are associated with liquefaction during earthquakes, such as ground oscillation, flow failure, loss of bearing capacity, lateral spreading, subsidence, and buoyancy. Buoyancy and lateral spreading are major critical effects for pipelines. A simplified scheme with possible induced mechanism is shown in Figure 1.

Thus, it is important to identify the liquefaction-prone areas for proper siting and design. The evidence of uplifted embedded structures induced by soil liquefaction was observed in numerous earthquakes, such as the 1964 Niigata Earthquake and the 1983 Nihonkai-Chubu Earthquake [1]; proofs of lateral spreading were found, for example, with the 1906 San Francisco Earthquake [2] and 1971 San Fernando Earthquake [3]. In these years, the interest in studying the phenomenon was developed and it boosted the research activities on liquefaction and related effects on buried structures. Liquefaction usually develops in case of predisposing factors such as water table at the ground surface; young, soft, and cohesionless soils; and uncompacted and poorly filling areas; it also develops in case of triggering factors such as strong ground motion with long duration. The effects of liquefaction on pipelines pose attention on three main aspects: the buoyancy that leads to pipelines damages at the boundary with manholes, the permanent ground deformation associated with liquefaction-induced 


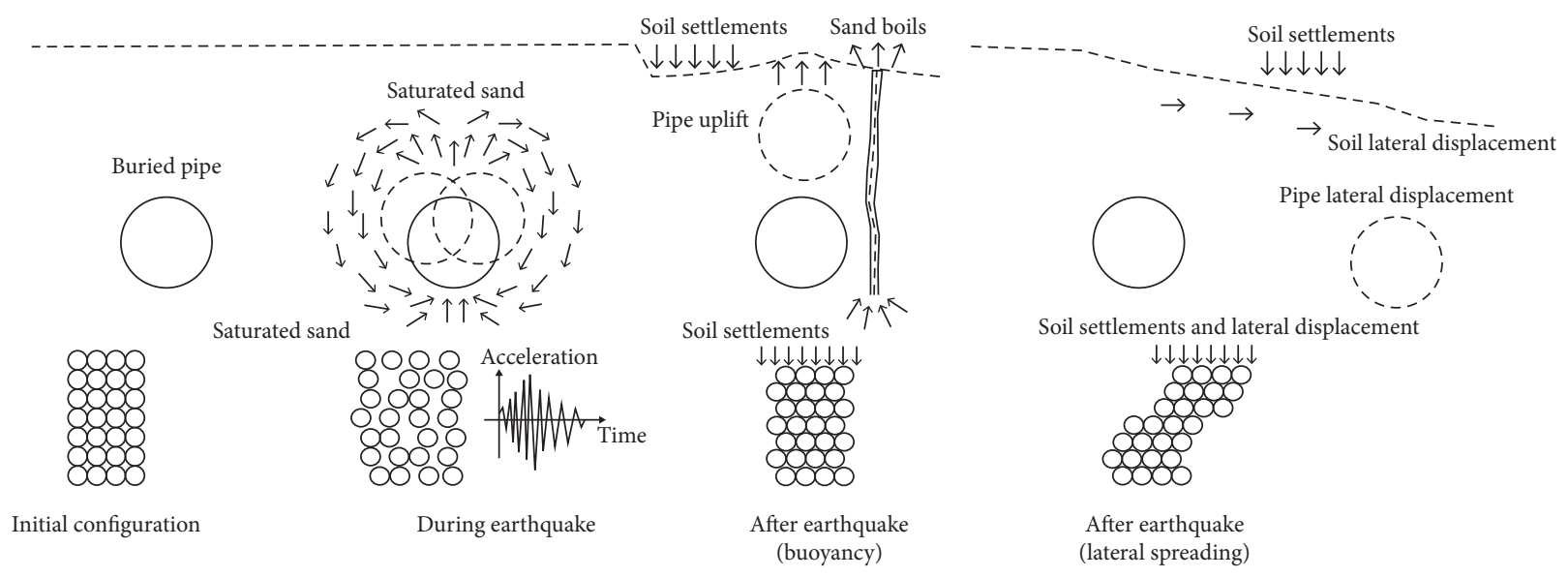

FIGURE 1: Simplified scheme of liquefaction-induced effects on buried pipelines.

lateral spreading, and the presence of nonuniform soil conditions that causes differential dynamic response effects at the boundaries between pipelines crossing liquefiable and nonliquefiable areas [4]. Buoyancy develops in areas with flat ground and groundwater table at the top surface, and when embedded structures are located in these sites, upward displacement is experienced. Lateral spreading develops on gentle slopes (i.e., 0.3-3 degrees) and is characterized by a movement of the liquefied surrounding soil that, in the presence of a buried structure, mobilizes the full passive pressure against it, thus resulting in a lateral movement of the pipeline as well. It is clear how both of the displacements can cause a pipeline breakage with negative consequences of functionality loss, leakage of the transported fluid with related effects, and the necessity of restoration works. Lateral spreading can be even more disruptive considering that the amount of horizontal displacement can be uncontrollable if liquefaction affects a large area. The adoption of ductile materials for pipelines could be not enough to face the large deformations imposed on underground structures. Moreover, lateral spreading can be difficult to identify due to this characteristic of gentle slopes. In the case of buoyancy, even if the liquefaction of surrounding soil involves a wide area, the maximum effect can be an upward displacement equal to the pipeline burial depth that exposes the structure above the ground surface. In most cases, pipelines are buried at shallow depths, so buoyancy is generally characterized by a maximum pipeline displacement of about $1.5 \mathrm{~m}$. Moreover, the lateral spreading of soil can induce different failure modes on pipelines depending on the crossing way of ground deformation with respect to the pipeline axis. A random crossing can simultaneously impose compression, tension, and bending on the pipe, perpendicular crossing generates bending stresses, and parallel crossing leads to failures for tension, compression, or local buckling [5]. In the study by O'Rourke and Lane [6], the liquefaction hazard and its effects on buried pipelines are widely described. In this paper, a state-of-the-art review for real observations of pipeline performances with reference to buoyancy and lateral spreading is provided together with the results achieved through the experimental and numerical modelling conducted on the specific issues and simplified analytical approaches to quantify the forces acting on the pipe for both uplift and lateral displacement are recalled. All these factors contributed to the accomplished level of knowledge and are fundamental to state the advances of the research about what has been clearly understood and what should be instead further assessed.

\section{Pipeline Performances Based on Real Observations}

2.1. Uplift and Lateral Spreading Case Histories. The uplift of buried pipelines induced by soil liquefaction was observed in several earthquakes such as the 1964 Niigata Earthquake, the 1983 Nihonkai-Chubu Earthquake [1], the 1993 KushiroOki Earthquake (due to surrounding backfilled sand and alluvial deposits) [7], the 1994 Hokkaido-Toho-Oki Earthquake [8], and the 2004 Niigata-ken Chuetsu Earthquake (due to surrounding refilled loose sand near sewage structures). For the latter earthquake, during the restoration work in Nagaoka City, a $20 \mathrm{~cm}$ diameter pipeline made of vinyl chloride and uplift of $50 \mathrm{~cm}$ were found, bent and pulled off in correspondence to a joint [9]. Damages of pipelines extensively occurred in the 2011 Tohoku Earthquake [10].

The 1906 San Francisco and 1989 Loma Prieta earthquakes liquefaction observations are summarized by Holzer [11], with an evaluation of pipeline damage. With the 1906 San Francisco Earthquake, an extensive damage to pipelines was found throughout the liquefaction areas in zones of deepest submerged artificial fill and along the boundaries of submerged fill where ground cracks and severe differential movements were observed. Consequent to the Loma Prieta Earthquake, analogous effects to those encountered during the San Francisco Earthquake, mainly with reference to the loose sandy fills around the margins of San Francisco Bay, and the natural deposits that lie beneath the valleys were observed, strengthening the vulnerability assessment of the areas. Indeed, liquefaction occurred almost exclusively within areas underlain by water-saturated, late Holocene alluvial and estuarine deposits. Soil liquefaction and liquefaction-induced lateral spreading resulted in considerable damages to lifelines, mostly in coastal and alluvial deposits and in uncompacted artificial fills. There were cases of fire 
spreading uncontrolled due to liquefaction-induced ground deformations that broke critical pipelines. In the study by O'Rourke et al. [12], it is reported that approximately $50 \%$ of all pipeline breaks from the 1906 San Francisco Earthquake occurred within zones of lateral spreading due to liquefaction.

O'Rourke and Palmer [13] described the earthquake performances of gas transmission pipelines and reported extensive pipeline damage in areas of liquefaction during the 1933 Long Beach Earthquake.

With the 1971 San Fernando Earthquake, 11 pipelines were found to cross the areas involved in landsliding and lateral spreading. Moreover, two high-pressure natural gas transmission pipelines were severely damaged by the soil displacements. A specific study on the damage of transmission pipelines produced by liquefaction-induced soil displacements was conducted by O'Rourke and Tawfix [14].

The 1983 Nihonkai-Chubu Earthquake caused extensive destruction of lifeline facilities and, in Noshiro City, buried gas, water, and sewage pipelines suffered damages associated with ground displacements triggered by liquefaction [15]. By comparing the magnitude of horizontal displacements and the damage to lifelines, a clear correlation between magnitude and extent of damage was highlighted, with the gas distribution network being most affected. Liquefaction widely occurred in Noshiro City, where a permanent ground displacement in a gentle slope was detected and embedded gas pipelines were damaged in the area with separation at the connections and bending at the curves [16]. O'Rourke and Lane [6] reported the study of a steel pipe transporting natural gas that showed cracks in five locations over $1.5 \mathrm{~km}$. The authors highlighted the cause to be in the compressive permanent ground displacement. The lateral spreading developed in a direction perpendicular to the pipeline axis, and the pipe was not able to sustain the bending deformation at the elbow. A case of pipeline buckling was also detected within the area of liquefaction-induced lateral displacement in Dagupan City $[17,18]$. Nishio [19] tried to investigate the failure mechanisms of continuous steel pipelines during the 1983 Nihonkai-Chubu Earthquake that seemed to be associated with the deformation process during the earthquake other than with the poor welding factor. Twelve damage failures of gas pipelines were investigated, and most of them were attributed to the repeated compressive and tensile ground deformations during the liquefaction rather than to the permanent deformation itself.

The 1994 Northridge Earthquake induced permanent ground deformations that affected the Potrero Canyon characterized by the presence of alluvial deposits within a depth of $80 \mathrm{~m}$. Sand boils and ground cracks were observed, and the latter were of both compressional and extensional types along the southern margin of the canyon, with few lateral displacements. In this scenario, pipelines experienced separations and cracks at the welds, most of them in correspondence to the ground cracks [20]. With the same 1994 Northridge Earthquake, Holzer et al. [21] described the damage to a steel pipe transporting gas, mainly consisting of tensile and compressive failures, respectively, in zones of tensile and compressive ground deformations. These ground deformations were located in an alluvial fan zone, and the ground deformation pattern led to attribute the ground movement to liquefaction, consequent to the reconnaissance on the site.

With the 1995 Kobe Earthquake, geotechnical failures were the main causes of damages including liquefaction effects. Two major and one small water treatment plants were damaged, and the distribution system was pulled away [22].

Ghayamghamian et al. [23] stated that during the 2007 Niigata-ken Chuetsu-Oki Earthquake, liquefaction was among the main geotechnical features that caused severe damage to lifelines.

The 2010-2011 Christchurch Earthquake sequence caused repeated liquefaction manifestations, and in areas close to the waterways, the liquefaction mainly caused lateral spreading. The widespread liquefaction in the suburbs of Christchurch, which interested almost one-thirds of the city area, shattered the lifelines to large areas resulting in numerous failures and reduction/loss of service for buried pipe networks [24]. Moreover, Cubrinovski et al. [25] highlighted a link between the liquefaction severity and the pipe network damage, observing $80 \%$ of the damages of the water network falling in liquefied areas. It was also stated that ductile materials and flexible pipe systems performed much better than concrete, steel, and iron pipelines with several fewer damages.

In the occurrence of the 2010 Chile Earthquake, the GEER report [26] summarized, among others, the soil liquefaction and lateral spreading deformation effects. The presence of a large number of rivers induced widespread liquefaction of loose saturated river sediments. Liquefaction effects with associated deformations had a strong engineering impact on the transportation corridors and buried structures were also damaged. There was a case of pipelines subjected to longitudinal strain due to the lateral spreading that may have caused the pipes distress.

2.2. Limitations. From the real observations, it is possible to make guesses on the nature of the damage, but experimental modelling and numerical modelling are fundamental to understand the mechanisms and confirm or discard the hypotheses. On the other side, without real observations, it would not be possible to validate laboratory test performances or numerical simulation results. These considerations highlight the importance of all these three factors in the understanding process.

Case histories are very useful to observe the performances of the analysed structures in different scenarios; however, if the pipeline after the earthquake and related liquefaction event does not expose above the ground and if the network does seem to be working properly, then it may be possible not to detect deformations related to liquefaction effects at the moment. In this context, buoyancy and lateral spreading effects can also be detected after years, when the area will need to be inspected for maintenance, renewal, or other operations. 
In case histories, it is difficult to observe the subsurface deformation. This limitation can be overcome with experimental simulations and is nowadays solved, thanks to the monitoring that can provide data on soil deformation as well. In the latter case, it appears evident that the monitoring is going to be localized in specific areas, so again, it is not possible to have a complete overview of the situation in the soil deposit. Despite this, field monitoring is a very precious source of knowledge to provide quantitative measurements of real performances that were only qualitatively provided in past years.

Another limitation of the real observations is the difficulty in extracting information about the different parameters affecting the problem in hand. Indeed, it may take years to address the influence of one single parameter on the pipeline response. This limitation can be solved by using numerical simulations through parametric analyses that allow exploring a huge variety of cases.

\subsection{Pipeline Performances Based on Experimental Modelling.} Experimental modelling to study the response of buried pipelines to the liquefaction phenomenon is usually conducted by using the shaking table or centrifuge devices. In the following, some of the experimental tests that were conducted over the years to address the pipeline performances are presented, with main findings and with the limitations of the used approaches and the state of knowledge. All the studies recalled here refer to the displacements induced by soil liquefaction and to the pipelines or underground structures experiencing similar behaviour.

2.4. Findings. Yasuda et al. [7] performed shaking table tests to study the mechanism of floatation, by using different soil relative densities (i.e., Toyura sand with $0 \%, 30 \%, 50 \%$, and $70 \%$ relative densities), two diameters (i.e., $6 \mathrm{~cm}$ and $55 \mathrm{~cm}$ ), and five different specific gravities $(0.5,1.5,1.7,1.8$, and 1.9) for the pipeline. The pipe was buried at a depth of $30 \mathrm{~cm}$. Horizontal shaking motion with $3 \mathrm{~Hz}$ frequency and $0.25 \mathrm{~g}$ acceleration amplitude, until the flotation stopped, was applied. Two different cases of water table were also considered: water at the top ground and water at $15 \mathrm{~cm}$ from the ground surface. Some tests were also repeated by increasing the acceleration amplitude to $0.4 \mathrm{~g}$. It was found that the speed of floatation decreased with the soil density, the pipe specific gravity, and the water level; in denser sand, the pipe did not rise to the ground surface. The speed of floatation decreased slightly with the diameter of the pipe. The speed of floatation in case of higher acceleration amplitude was higher as well. The shaking table tests were also conducted to study the effects of the backfill on the flotation. A soil relative density of $30 \%$ was used for the backfill, while a pipe with a diameter of $11.4 \mathrm{~cm}$ with 0.75 specific gravity was tested. It was observed that the width and the depth of the backfilled trench affect the floatation of the pipe; the speed and the maximum height of floatation increased with both the width and the depth of the backfill. Floatation can also occur if liquefaction affects the backfill only, and it depends on the dimensions of the backfilled trench, as already mentioned.
The effect of permeability at the boundary between the trench and surrounding soil is also an important factor.

Exhaustive research on the uplift behaviour of underground structures induced by soil liquefaction was conducted by Koseki et al. [27] by performing shaking table tests. The authors described the uplift as the result of three combined mechanisms, such as the reduction of soil strength that makes deformations possible in the surrounding soil, the generation of a horizontal movement of pore fluid due to the presence of the pipe, and the reconsolidation process after excess pore pressure dissipation. Already from this research, the development of a cavity during the uplift process, immediately below the pipe, was highlighted.

Sasaki and Tamura [28] performed centrifuge tests to evaluate the underground structure performances in uplift displacement induced by soil liquefaction. For the experiments, the centrifuge provided by the Public Works Research Institute in Japan was used, under a centrifugal acceleration of $50 \mathrm{~g}$. The results of the tests showed that the uplift starts after liquefaction is triggered and continues until the shaking is ongoing. Some of the test results are extracted from the original work and summarized in Table 1 to support the comments provided here. The case of an underground structure model with $5 \mathrm{~m}$ width, $3.75 \mathrm{~m}$ height, and an apparent density of about $8 \mathrm{kN} / \mathrm{m}^{3}$ is considered. It is buried in a soil deposit prepared by air pluviation with Toyoura sand, with groundwater level at the top surface. As input motion, a sinusoidal waveform of 20 cycles and $1.2 \mathrm{~Hz}$ frequency and the Kobe Earthquake are considered. The authors observed that the application of a sinusoidal input motion leads to a bigger uplift than a real earthquake input, as it can be stated by comparing the cases 98-02 and 98-04. Moreover, the uplift was found to be higher in case of a higher amplitude of the shaking (comparison between cases 97-02 and 98-02), looser soil condition (98-04 and 98-05), and a lower ratio between the structure width, $W$, and the depth of the liquefiable layer below, $D$ (cases 01-03 and 0105). Finally, by looking at the ground deformations, it was stated that the structure and the soil above move upward, while the soil beside moves downward, towards the bottom of the structure, as it can be explained by the different overburden pressures below the structure and on the lateral side.

The failure mechanism involved in the uplift of pipelines in liquefied soils was explained by Stringer and Madabhushi [29]. Tests were conducted with a $1 \mathrm{~g}$ shaking table with a soil container of dimensions $0.4 \mathrm{~m} \times 0.2 \mathrm{~m} \times 0.5 \mathrm{~m}$ filled with Hostun S28 sand with $36 \%$ relative density. The tunnel has a diameter of $0.5 \mathrm{~m}$ that is 100 times smaller than the prototype, and it is installed with the crown at the top surface. Three input motions were applied to the model with maximum accelerations of $0.65 \mathrm{~g}, 0.71 \mathrm{~g}$, and $1.02 \mathrm{~g}$, resulting in uplift displacements of $4.21 \mathrm{~mm}, 3.90 \mathrm{~mm}$, and $1.62 \mathrm{~mm}$, respectively. Thanks to image acquisition analyses, the generation of soil flow around the pipe was generated, with the soil on the lateral sides moving below while the pipe is displaced upward. The research was focused on the response in uplift with reference to the time history, and it was pointed out that the vertical displacement of the pipe is 
Table 1: Comparisons on uplift displacements for different test conditions (extracted from [28]).

\begin{tabular}{|c|c|c|c|c|c|}
\hline Case & Relative density (\%) & Ratio W/D (-) & Wave type & Shaking acceleration $(\mathrm{g})$ & Uplift (m) \\
\hline $97-02$ & 80 & 1.33 & Sinusoidal & 0.294 & 0.23 \\
\hline $98-02$ & 80 & 1.33 & Sinusoidal & 0.785 & 0.72 \\
\hline $98-04$ & 80 & 1.33 & Kobe & 0.785 & 0.26 \\
\hline 98-05 & 50 & 1.33 & Kobe & 0.785 & 0.60 \\
\hline $01-03$ & 50 & 0.80 & Sinusoidal & 0.294 & 1.32 \\
\hline $01-05$ & 50 & 1.33 & Sinusoidal & 0.294 & 1.21 \\
\hline
\end{tabular}

characterized by a frequency that is double of the input motion frequency; moreover, it was found that the pipe response to the input motion is composed of the repetition of the mechanism observed in half loading cycle. The half loading cycle can be divided into three phases, where the first phase shows no movements, the second phase is composed of both uplift and lateral displacement of the pipe, and the last phase consists of downward displacement. This means that in one loading cycle, there are only two phases in which uplift sums up. The uplift proceeds at a constant rate during the shaking and ceases when the shaking ends, as it happens for the horizontal displacements as well.

Ichii et al. [30] identified the uplift rate to be a good parameter to be correlated with the total amount of uplift. From the results of shaking table tests, the importance of the fine content of the surrounding material and the insignificance of permeability on the uplift velocity were discovered. The explanation should be found in the stress-strain behaviour of the soil, and the velocity of uplift can be related to the sand recovery of strength linked with dilatancy. In the experiments, a $60 \mathrm{~mm}$ diameter pipe was used, placed in the middle of a sand layer $200 \mathrm{~mm}$ deep and $400 \mathrm{~mm}$ wide. The sand layers were built up in underwater conditions and compacted by hand. Sinusoidal input shaking of $10 \mathrm{~Hz}$ for 4 or $5 \mathrm{~s}$ with $0.4 \mathrm{~g}$ amplitude was applied. For small relative density (i.e., $30 \%$ ), the uplift rate was not constant, increased rapidly at the beginning of the shaking (about $25 \mathrm{~mm} / \mathrm{s}$ at $0.5 \mathrm{~s}$ ), decreased later (about $13 \mathrm{~mm} / \mathrm{s}$ at $1.5 \mathrm{~s}$ ), and increased again at the end (it reached $50 \mathrm{~mm} / \mathrm{s}$ at $4 \mathrm{~s}$ with a final uplift of $100 \mathrm{~mm}$ ). For higher relative density (i.e., 50\%), this effect is only slightly observed $(7 \mathrm{~mm} / \mathrm{s}$ at $1 \mathrm{~s} ; 4 \mathrm{~mm} / \mathrm{s}$ at $3.5 \mathrm{~s}$; and $5 \mathrm{~mm} / \mathrm{s}$ at $5 \mathrm{~s}$ with a total uplift of $25 \mathrm{~mm}$ ). Some tests were also conducted to investigate the effects of fine contents by adding crushed-stone powder to the sand layer. In this case, methylcellulose in the pore fluid was used for the permeability similitude. For noncohesive fine contents (FCs) from $0 \%$ up to $10 \%$, the uplift velocity was found to be increasing with increasing FC. For FC exceeding a certain amount (i.e., $20 \%$ ), the uplift behaviour changed with a very small rate at the beginning of the shaking and the upward displacement also continued after the shaking until the pipe reached the ground surface.

Chian and Madabhushi [31] prepared ground models using Hostun and Fraction $\mathrm{E}$ sand at a relative density of $45 \%$. They employed the beam centrifuge in Cambridge for the experiments. A circular hollow structure was used with different diameters and buried at different depths. Multiple earthquake motions were inputted. Some characteristics are summarized in Table 2, while other details can be found in
Table 3 together with the uplift results. The outcome of the research was that, overall, lower burial depths or smaller diameters for pipelines lead to higher uplift displacements. The authors computed two effect ratios for burial depth and diameter to allow comparisons of uplift in different test conditions. Both ratios are based on the vertical equilibrium of forces and multiplied by a modification factor that accounts for multiple aspects involved in the phenomenon. The depth effect ratio is affected by the difference in the overlaying soil thickness, in the length of the shear planes, in the excess pore pressure, in the soil densification after an earthquake, in the confining stress, and in the soil permeability. The diameter effect ratio is affected by the difference in the excess pore pressure, in the soil densification, and in the soil stiffness near the surface. Based on the same centrifuge tests, Chian and Madabhushi [32] further discussed that the grain size distribution affects the uplift, thus showing smaller entity of uplift for finer sand due to smaller excess pore pressure generation. This can be seen by comparing results between Hostun and Fraction $E$ sand, and indeed, the measured uplifts in DC-03 are about half the values in DC-02 in both first and second earthquake loadings. The hydraulic conductivity of the sand is apparently not directly related to the uplift displacement, but it can be due to different effects of grain size and fluid migration interacting and cancelling out each other. Finally, in general, a higher relative density of soil deposit after a first earthquake reduces the pipe uplift for a second earthquake. However, in case of a second earthquake, if it is characterized by a longer duration, uplift can be even more. In this case of second earthquake occurrence, the structure cover is going to be less too, because of the previous pipe uplift and the densification of soil, thus making the uplift easier in terms of force balance.

Chian et al. [33] studied the soil deformation during seismic pipe uplift in liquefiable soil through centrifuge and full-scale model tests using the Particle Image Velocimetry (PIV) technique. The soil flow that was already introduced by Stringer and Madabhushi [29] was confirmed by the results of this research. The soil deformation consists of flow around the pipe that can be described accounting for three components. The soil above the pipe moves upward due to the pipe displacement; the soil lateral to the pipe moves downward and is continuously partially substituted from the soil above; and the soil at the bottom of the pipe coming from the soil laterally to the pipe moves towards the base of the structure while it uplifts and a cavity is generated. The cavity is quickly filled by the soil so that the pipe cannot move back downward. An effect of dilation of the soil close 
TABLE 2: Tests characteristics (extracted from [31]).

\begin{tabular}{|c|c|c|c|c|c|}
\hline Test & Pipe & & Soil & & Container \\
\hline ID & Mass $(\mathrm{kg})$ & Sand type & $\begin{array}{l}\text { Pore fluid viscosity/centrifuge } \\
\text { g-level (cSt/g) }\end{array}$ & Hydraulic conductivity $(\mathrm{m} / \mathrm{s})$ & Box type \\
\hline DC- 02 & 5425 & Hostun & 1 & $4.37 \times 10^{-4}$ & ESB box \\
\hline DC-03 & 5425 & Fraction $\mathrm{E}$ & 1 & $0.99 \times 10^{-4}$ & ESB box \\
\hline DC-04 & 5425 & Hostun & $1 / 9$ & $3.93 \times 10^{-3}$ & ESB box \\
\hline DC- $04 b$ & $5425 \& 438$ & Hostun & $1 / 3$ & $1.31 \times 10^{-3}$ & Window box \\
\hline DC-07 & $5425 \& 438$ & Hostun & 1 & $4.37 \times 10^{-4}$ & Window box \\
\hline DC- 10 & $2411 \& 195$ & Hostun & 1 & $4.37 \times 10^{-4}$ & Window box \\
\hline
\end{tabular}

TABLe 3: Experimental uplift displacement (extracted from [31]).

\begin{tabular}{|c|c|c|c|c|c|}
\hline \multirow{2}{*}{$\begin{array}{l}\text { Test } \\
\text { ID }\end{array}$} & \multicolumn{2}{|c|}{ Earthquake } & \multicolumn{3}{|c|}{ Pipe } \\
\hline & Peak bedrock acc. (g) & Duration (s) & Diameter $(\mathrm{m})$ & Depth to axis/diameter $(-)$ & Uplift (m) \\
\hline DC-02 & 0.22 & 25 & 5.00 & 1.5 & 0.676 \\
\hline DC-02 & 0.22 & 25 & 5.00 & 1.1 & 0.816 \\
\hline DC-02 & 0.23 & 40 & 5.00 & 1.5 & 0.754 \\
\hline DC-02 & 0.23 & 40 & 5.00 & 1.1 & 1.512 \\
\hline DC-03 & 0.22 & 25 & 5.00 & 1.5 & 0.337 \\
\hline DC-03 & 0.22 & 25 & 5.00 & 1.1 & 0.436 \\
\hline DC-03 & 0.23 & 40 & 5.00 & 1.5 & 0.264 \\
\hline DC-03 & 0.23 & 40 & 5.00 & 1.1 & 0.447 \\
\hline DC-04 & 0.21 & 25 & 5.00 & 1.5 & 0.559 \\
\hline DC-04 & 0.21 & 25 & 5.00 & 1.1 & 0.894 \\
\hline DC-04 & 0.22 & 40 & 5.00 & 1.5 & 0.286 \\
\hline DC-04 & 0.22 & 40 & 5.00 & 1.1 & 0.687 \\
\hline DC- $04 b$ & 0.08 & 25 & 5.00 & 1.5 & 0.143 \\
\hline DC-04b & 0.08 & 25 & 1.42 & 1.5 & 0.154 \\
\hline DC- $04 b$ & 0.09 & 40 & 5.00 & 1.5 & 0.018 \\
\hline DC- $04 b$ & 0.09 & 40 & 1.42 & 1.5 & 0.096 \\
\hline DC- $04 b$ & 0.09 & 50 & 5.00 & 1.5 & -0.022 \\
\hline DC- $04 b$ & 0.09 & 50 & 1.42 & 1.5 & 0.357 \\
\hline DC-07 & 0.09 & 25 & 5.00 & 2.0 & 0.025 \\
\hline DC-07 & 0.09 & 25 & 1.42 & 2.0 & 0.269 \\
\hline DC-07 & 0.10 & 40 & 5.00 & 2.0 & 0.027 \\
\hline DC-07 & 0.10 & 40 & 1.42 & 2.0 & 0.110 \\
\hline DC-10 & 0.17 & 18 & 3.33 & 1.5 & 0.182 \\
\hline DC-10 & 0.17 & 18 & 0.95 & 1.5 & 0.193 \\
\hline DC-10 & 0.18 & 25 & 3.33 & 1.5 & 0.058 \\
\hline DC- 10 & 0.18 & 25 & 0.95 & 1.5 & 0.236 \\
\hline DC-10 & 0.18 & 36 & 3.33 & 1.5 & 0.013 \\
\hline DC-10 & 0.18 & 36 & 0.95 & 1.5 & 0.289 \\
\hline
\end{tabular}

to the pipe is responsible for this movement of soil towards the cavity. The authors highlighted, in this process of uplift, the importance of the horizontal displacement of the pipe too. This can, indeed, be even larger than the upward displacement, thus stating the necessity to refer to a $2 \mathrm{D}$ movement other than a $1 \mathrm{D}$ problem.

The first author of this review article [34] performed shaking table tests to evaluate the performances of buried pipelines to soil liquefaction-induced vertical displacements and the benefit of possible remediation techniques. The study mainly focused on the response in the uplift of the pipeline subjected to a shaking sequence by changing the amplitude of sinusoidal input motion in subsequent steps. The amount of uplift is bigger for the first shaking step that induces complete liquefaction of the soil model, and the uplift continues with the next shaking steps until the shaking sequence is overall able to liquefy some of the layers close to the pipe. For these next shakings, the amount of uplift is reduced, considering the increase in relative density of the soil deposit due to the previous shaking history. Different apparent unit weights of the pipe were also investigated, and it was found that pipelines with an apparent unit weight smaller than the water unit weight undergo uplift as expected. In this case, uplift starts even if the excess pore water pressure development is not high enough to cause liquefaction, due to the light weight of the pipe and the water pressure increase. For a pipe with an apparent unit weight similar to the dry sand unit weight, the pipe can slightly displace upward depending on the relative density of the soil deposit. A pipe with apparent unit weight slightly bigger than the saturated sand unit weight experiences settlements that are lower than the free-field ground settlements. The 
investigated model is in scale $1: 10$, and the soil deposit is $2.8 \mathrm{~m}$ long, $0.4 \mathrm{~m}$ wide, and $0.55 \mathrm{~m}$ high, prepared with Silica sand \#7 poured with a hopper. The pipeline is in PVC with an external diameter of $11.4 \mathrm{~cm}$ and installed at a laying depth of $25 \mathrm{~cm}$ from the ground surface. The earthquake sequence is composed of twelve shaking steps with changing amplitude, and each step is characterized by sinusoidal waves of $5 \mathrm{~Hz}$ frequency and $4 \mathrm{~s}$ duration. The steps are run subsequently, immediately after the dissipation of the excess pore water pressure generated from the previous step. The test results to support the considerations recalled here are listed in Table 4. For test $\# 1$, the soil is prepared with $50 \%$ relative density and the pipe has an apparent unit weight of $6.8 \mathrm{kN} / \mathrm{m}^{3}$; for test \#2, values are, respectively, $40 \%$ and $13.2 \mathrm{kN} / \mathrm{m}^{3}$; and for test \#3, values are, respectively, $50 \%$ and $21.0 \mathrm{kN} / \mathrm{m}^{3}$.

Trautmann and O'Rourke [35] performed an experimental study to evaluate the response of buried pipelines to lateral ground displacement resulting from the relative movement between the pipeline and surrounding soil in a plane-strain condition. Force-displacement results of the lateral loading test showed that the displacements corresponding to the maximum force are substantially affected by the soil density. For shallow pipes, in loose sand, a lateral displacement bigger than $0.13 \mathrm{H}$ (with $\mathrm{H}$ depth to the pipe center) was necessary to attain the maximum force; for medium sand, a displacement of about $0.08 \mathrm{H}$ achieved the maximum force asymptotically; and for dense sand, the peak force was reached for a displacement of $0.03 \mathrm{H}$. However, referring to typical burial depths of pipelines, at large displacements, the residual force is only slightly affected by the density, being similar for loose sand and medium sand at different tested depths. This similar residual force is explained by the compaction of loose sand due to the lateral movement of the pipe that increases the local density and shows a soil resistance that is comparable with that of medium sand. Moreover, it was observed a small influence of the pipeline surface roughness on the system response. Finally, the force-displacement behaviour by varying the pipeline depth/diameter ratio was analysed. It was found that for a depth/diameter ratio smaller than 8 , the maximum and residual forces are the same for loose sand and medium sand and the residual force is the same for both dense sand and medium sand; for a depth/diameter ratio between 2 and 8 , the residual force for dense sand is larger than that for loose sand and medium sand, and for a depth/diameter ratio bigger than 8 , the residual force for dense sand becomes gradually larger than the maximum force for medium sand. The reasons can be found in the soil dilatancy and arching at depth effects. Considering that the soil/pipe displacements vary along with the longitudinal development of the pipeline, the maximum force is not exercised for the whole length. In the case of large displacements, the residual forces dominate the problem of pipeline response, and if the depth/ diameter ratio in smaller than eight, then the surrounding soil density does not affect the residual force.

Towhata et al. [36] performed shaking table tests to make considerations on the drag force necessary to pull a buried pipeline laterally. This study was conducted by referring to three test conditions: dry sand subjected to strong shaking; water-saturated conditions corresponding to a loose liquefiable sandy ground; and extremely loose sand. The latter tries to keep the model in the same dilatancy expected for the sand in the real field. As a result of the first condition, the drag force was found to be lower when the magnitude of shaking is higher and it reduces the sand shear strength. The amplitude of the shaking also affected the drag force in the second and third condition of the water-saturated model ground, in a way that varies with the pipe movement velocity and that becomes more evident in case of completely liquefied sand, thus indicating an apparent viscous behaviour. Moreover, in case of strong shaking, the drag force during the shaking was found to be smaller than that after the shaking, and the small value of drag force was attributed to undeveloped effects of dilatancy due to the big amplitude shaking.

Ashford and Juirnarongrit [37] evaluated the response of single piles and pipelines to liquefaction-induced lateral spreading by using controlled blasting in two full-scale experiments. The first lateral spreading experiment setup is composed of a test site of $25 \mathrm{~m}$ wide and $100 \mathrm{~m}$ long, with sheet pile quay wall installed on the left short side of the site and anchored to tied rods fixed at $\mathrm{H}$-piles to avoid movements of the wall. On the front end was located a waterway. From about $25 \mathrm{~m}$ away from the wall, the ground surface started to slope upward with an inclination of $4 \% .19 \mathrm{~m}$ away from the wall, a single pile of $318 \mathrm{~mm}$ diameter is installed, and other similar free-head single piles were located nearby. The response of these piles was monitored during the tests, but here, it is mainly focused on the response of the pipelines. One natural gas pipeline of diameter $500 \mathrm{~mm}$ and one electrical conduit of diameter $267 \mathrm{~mm}$, both $25 \mathrm{~m}$ long, were installed about $30 \mathrm{~m}$ away from the quay wall at a depth of $1.75 \mathrm{~m}$. These two pipelines were located transversally to the direction of the flow and with both extremities anchored to the sheet pile wall. One additional natural gas pipeline of $500 \mathrm{~mm}$ diameter and length of $22 \mathrm{~m}$ was installed parallel to the direction of the flow, $1 \mathrm{~m}$ below the ground surface and with only one end anchored to the sheet pile wall. Blast holes were organized in squared grid mesh and charges were installed at different depths. The explosives had the scope to loosen the soil and allow the free flow of soil. In the last part of the test, supplementary explosives were used to break the tied rods of the quay wall so that its free movement could generate additional soil flow in the investigated area. The second lateral spreading experiment setup is the same coming after the execution of the first test, only quay wall and sheet piles around were removed, the waterway was excavated on one end of the test site, and the slope was steeper than the previous case. This second experiment was conducted to attempt the possibility of inducing a higher level of soil deformations and further assess the piles and pipeline performances. With the first test, a similar movement of both transverse pipelines with maximum displacement was observed in the middle of the pipelines. In the second test, the movement of both transversal conduits was half of those measured with the first test and this was attributed to less liquefaction 
Table 4: Pipe vertical displacements summary (extracted from [34]).

\begin{tabular}{|c|c|c|c|c|c|c|c|c|c|c|c|c|}
\hline \multicolumn{13}{|c|}{ Shaking step (No.) } \\
\hline & 1 & 2 & 3 & 4 & 5 & 6 & 7 & 8 & 9 & 10 & 11 & 12 \\
\hline \multicolumn{13}{|c|}{ Base acceleration amplitude (g) } \\
\hline & 0.1 & 0.2 & 0.3 & 0.4 & 0.5 & 0.6 & 0.5 & 0.4 & 0.3 & 0.2 & 0.1 & 0.8 \\
\hline$\#$ & \multicolumn{12}{|c|}{ Vertical displacement (mm) (-, uplift; +, settlement) } \\
\hline 1 & 0.3 & -1.8 & -10.5 & -6.6 & -4.7 & -7.6 & -6.2 & -5.1 & -1.5 & -2.5 & -0.7 & 4.0 \\
\hline 2 & 1.0 & -1.0 & -3.8 & -1.1 & -0.6 & -2.3 & -0.7 & -0.4 & -0.1 & 0.1 & 0.1 & 2.2 \\
\hline 3 & 0.9 & 0.7 & 2.7 & 4.0 & 4.0 & 4.2 & 3.1 & 2.2 & 0.9 & 0.5 & 0.0 & 0.3 \\
\hline
\end{tabular}

susceptibility. The scope of the test for transverse pipelines was to investigate the bending response induced by the soil flow, while the test for the longitudinal pipeline was to evaluate its performance to axial frictional forces imposed by the soil moving with respect to the pipeline. It was found that the strain distribution was nonuniform along the pipe length due to blasting-induced nonuniform pressure from the compression waves in the surrounding soil. Due to the difference in diameter of the two transversal pipelines and the same experienced movement, the strain distribution was smaller for the smaller diameter pipe. For the case of a longitudinal pipeline, the maximum movement was found to occur in the middle of the pipe. This was associated with soil movement produced by the blasting, which caused the pipeline to move significantly in transversal direction rather than in the longitudinal direction. Moreover, a significant strain at the top and the bottom of the pipeline was recorded due to vertical movements (i.e., bending strain), which seemed to be more important than the frictional force expected to be analysed.

Shimamura et al. [38] investigated the transverse horizontal load imposed on buried pipelines by liquefaction-induced permanent ground deformations. Previous research studies on transverse horizontal loads on the pipeline were conducted by using static model ground with application of force to displace the pipe. In this research, instead, dynamic centrifuge model tests were used, with pipelines buried in nonliquefiable soil layers immediately above the liquefiable layers. Two cases of ground area were analysed, i.e., behind a quay wall and sloping ground area. The loads obtained in both tests of the quay wall and sloping ground were smaller than the loads obtained by displacing the pipeline with an applied force in a static ground model. The ratio between the maximum load in the quay wall model test and the maximum load in the pipe movement test was in the range $0.25-0.34$. Instead, the ratio between the maximum load in the sloping ground model test and that obtained in the pipe movement test fell within the range $0.31-0.44$. This finding was further substantiated by analysing the load-ground displacement curves before and after the end of the shaking, which showed continuity, thus discarding the shaking effects as a cause for the decrease in load. Another trial was made, using an upper layer with the same relative density as the soil layer below, and the maximum load was found to be similar to the test with higher density upper level, thus leading to the possibility that a maximum load equal to the residual strength of the sand is exercised on the pipe. Cracks were observed after the test on the front side area of the pipe that could have decreased the confining pressure of the ground around the pipe, thus decreasing the load. In the same way, the tensile strain generated in the ground could have decreased the confining pressure and reduced the load.

Sarvanis et al. [39] evaluated the soil-pipe interaction in axial and transverse directions. In the first case, the principle is that the soil resistance develops due to the friction at the soil-pipe interface. In the case of transverse direction, the soil-resistant mechanism that governs the problems of strike-slip fault or landslide is reproduced. The results of the first case show the importance of soil dilatancy on the axial soil-pipe interaction, while for the transverse direction, the residual value of the sand internal friction angle governs the soil-pipe interaction response.

2.5. Limitations. The pipeline floatation is experimentally well understood, thanks to the exhaustive model tests that have been conducted to address the specific issue. The pipeline performance in terms of uplift, within the framework of soil liquefaction phenomenon, is assessed in terms of both involved mechanisms and affecting parameters.

Different is the case of the lateral spreading phenomenon, for which experimental modelling on the specific matter is not directly available for the evaluation of pipeline performances. This means that, to account for the effects of possible permanent ground displacement on the pipeline caused by soil liquefaction induced lateral spreading, it is usually referred to (1) analyses conducted on piles; (2) pull-out tests with statically applied forces; or (3) permanent ground displacements induced by fault ruptures or landslides. In the latter point, the replication of the ground deformation effects on pipelines, which is similar to that induced by fault movement or landslide in terms of tensional state, other than the phenomenon itself, is commonly adopted. This is done due to the difficulties of reproducing the lateral spreading phenomenon with embedded structure interaction in model testing. It is clear, however, that the stress-strain behaviour of soil undergoing liquefaction process is completely different from the application of a force that can only simulate the direction and the entity of displacement.

The blasting in the full-scale testing site was proven to successfully liquefy the soil and induce lateral spreading; however, the movement produced by the blasting is not 
controlled and can lead to different distributions of displacements and strains. It should be carefully used to assess performances that are related to earthquake-induced effects due to the differences in the generation of waves. The explosive loadings are used to address different pipeline performances as well. For example, Zhang et al. [40] studied the buckling response of the buried steel pipe under the effects of multiple explosive loadings.

The overall performances of buried pipelines are well caught from these model tests. However, being a reproduction in a laboratory of real mechanisms, some limitations can affect the results and impose restrictions on the extension of the achieved outcome to the real field. Moreover, it is worth considering that test results are dependent on the specific model test characteristics (e.g., model scale, similitude laws, geometries, model preparation, ground characteristics, and input motion characteristics) and, in most cases, it is difficult to generalize them, allowing quantitative comparisons.

Liquefaction is a very important issue but also challenging and still with open questions due to its complexity, mostly for the interaction with structures. In model tests, the liquefaction is one of the nonfully reproducible phenomena through the similitude theories. Big models should be used, to avoid scaling factors, but this would be very expensive. However, the possibility to use model tests depends on the specific results to be extracted from the experimental tests.

1-g shaking table tests are widely used to study the soilstructure interaction and liquefaction process development. However, the gravity field does not allow the reproduction of the confining pressure as in the real case. This stress level difference affects the strain level that alters the soil dynamic properties, and the reproduction of the stress-strain behaviour of sand is fundamental for describing the development of the liquefaction process. Moreover, there is often a difference related to the shaking frequency effects. These differences between the model and prototype also affect the similitude of residual displacements that govern the seismic damage in geotechnical structures, as it can be the case of pipeline residual displacements. Some scaling factors can be adopted to reduce the discrepancies, but the scaling factors cannot satisfy all the similarities with the real field.

Concerning, instead, the centrifuge devices, the centrifugal effect itself allows the respect of stress level even in a small-scale model, thus being able to reproduce the same stress-strain behaviour of soil in the real field. However, the limitation of this model testing can be found in the nonreproduction of geological history and ageing effects, as can be expected in model grounds prepared in the laboratory. Of course, in the real field, the soil deposit is affected, among others, by the geological history, the age, the soil grain packing, and the previous seismic shaking sequence.

Other than for the similarities, that would prefer the centrifuge device for the stress level correspondence, 1-g shaking table tests are usually adopted due to the easiness in preparing the models that have a smaller size than centrifuge models, other than smaller maintenance costs.

A deep analysis of the difference between 1-g shaking table tests and centrifuge modelling and the scaling factors to be adopted for the similitude law with the real field is provided by Towhata [41].

\section{Pipeline Performances Based on Numerical Modelling}

This section mainly refers to the numerical approaches adopted in the literature to investigate the response of embedded pipelines against liquefaction-induced displacements, focusing on the studies that addressed the problem from a computational point of view and analysing both the adopted strategies and solutions developed to overcome the difficulties linked to the modelling process. With reference to liquefaction-induced buoyancy and lateral spreading, an overview of the numerical simulations performed to assess the pipeline response against the abovementioned liquefaction effects is presented.

3.1. Findings. Pipelines need to be designed in order to guarantee their stability against both static and dynamic loads. Although the static calculation strategies are quite shared in the standard of practice, different approaches are used to evaluate the response of the embedded structures to dynamic excitations. The earthquake-induced effects along a pipeline can be modelled, applying to the structure a deformation pattern that is independent of the structural stiffness if the structure is softer than the soil. However, when the stiffness of both soil and structure is similar, great significance is assumed by the definition of the soil-structure interaction. The most convenient way to face this problem consists of avoiding to define the nondefinition of a proper interaction by imposing that pipeline moves in accordance with the surrounding soil [42], while different strategies are available if this interaction is considered. Thus, it is much more complicated when liquefaction needs to be accounted for. As clearly demonstrated by both the case histories and experimental tests, liquefaction manifests its effects on pipelines through permanent displacements, namely, permanent ground deformation (PGD), to be distinguished from transient ground deformation (TGD), which is mostly due to the seismic wave propagation. In order to predict the pipeline liquefaction-induced displacement, a useful preliminary tool to be used in the design process could be represented by the simplified methodologies that, worth reminding, are developed under specific conditions, and consequently, they are reliable only when these conditions govern the problem.

Even if a wide research body was developed in order to analyse the behaviour of tunnels in liquefiable soils (see, for instance, Chang et al. [43]; Zhuang et al. [44]; Liu and Song [45]) or the pipeline response crossing active faults (cf. Kouretzis and Bouckovalas [46]; Karamitros et al. [47]; Karamitros et al. [48]), a few numerical simulations that specifically aim at investigating the behaviour of pipes against liquefaction-induced lateral spreading and uplift were developed over the years. Two pivotal aspects, to be carefully analysed in order to guarantee the reliability of the numerical analysis and subsequent findings, are both the definition of the soil constitutive model and the soil-pipeline interaction that, consequently, must be chosen and checked 
carefully. Moreover, the definition of the pipeline material constitutive law assumes the same relevance, but it is beyond the scope of the study. In this framework, a key role is played by the solution strategy as well. Indeed, the most widespread computer software programs adopt the Finite Element or the Finite Difference Method. Software programs employing poorer strategies such as the Finite Volume Method or Material Point Method were, instead, rarely encountered.

Regarding the finite element strategy, Datta [42] performed an overview of the most adopted models where the soil-structure interaction is considered carrying out an evaluation of how they can be used to simulate the propagation of seismic waves. The following options were addressed: (i) beam on elastic springs; (ii) shell model for large diameter buried pipelines; (iii) plane-strain model of the structure; and (iv) hybrid model. Sarvanis et al. [39] identified two different approaches to the problem of buried pipelines subjected to PGD: the less-refined method of modelling the pipe as a beam element while nonlinear springs are deployed to describe the soil behaviour and a more advanced method that defines the pipe with shell elements and the soil with solid elements. In addition, Karamitros et al. [44] proposed an approach that weaves the main features of both methodologies, by considering shell elements to define the pipe behaviour and nonlinear spring elements to describe the soil constitutive behaviour.

O'Rourke and Lane [6] were among the first to use the finite element approach to study lateral spreading problems related to lifelines. They performed $2 \mathrm{D}$ parametric analyses using the finite element in-house software UNIPIP on steel water pipelines, in order to evaluate the pipeline sensitivity against maximum liquefaction-induced displacement, the width of the slide where this displacement takes place, and soil characteristics of the displaced mass. In their simulation, it was assumed that the pipeline intersects the soil mass perpendicularly to the instability plane. Only horizontal displacements were allowed, to evaluate slide effects, by defining a symmetric pattern that affects the pipe movements, as to reproduce the lateral spreading phenomenon. Furthermore, the pipe cross section is constrained against any wrinkling and ovaling of the pipe wall and only bend is allowed. The analysed pipeline is a continuous girth welded pipeline made of X-60 grade steel. Soil-pipeline interaction was modelled through the bilinear relationship as in Trautmann and O'Rourke [35], to define the transverse horizontal soil reaction, considering a secant horizontal stiffness. To catch this behaviour, spring-slider elements were adopted. As a result, it emerged that a closely bilinear relationship relates pipeline strains and maximum displacement of soil. In addition, when plastic flow initiated, an increase in tensile strain is observed, with a contemporary decrease in compressive strain, while a greater length of the pipeline is subjected to deformation as the structure displaces because of soil-structure interaction. Furthermore, the lateral spread width has a higher influence on pipeline strain compared to soil density and shear strength; indeed, it was found that values lower than $20 \mathrm{~m}$ can lead to high plastic strains. Finally, the research reveals that a key role to predict the deformation process is played by geomorphologic conditions.

Takada et al. [49] exploited the hybrid model to investigate the response of buried pipes under lateral spreading displacements. The authors aimed at validating a practical method to estimate lateral spreading-induced displacements on pipes. The finite element software ABAQUS was adopted in the study, and both slope and revetment deformation patterns were analysed. Three width measurements of the liquefied ground were considered based on the observations available after the 1995 Hyogoken-Nanbu Earthquake: 20 m, $100 \mathrm{~m}$, and $200 \mathrm{~m}$. The finite element model of the pipe was developed using 4-node shell elements implemented in ABAQUS, supported by inelastic springs to model the soil. The results suggested that plastic hinges can be triggered in three different points near the bend. The model is calibrated based on full-scale experiments, and satisfactory accordance in the response is shown.

Shao [50] performed a 3D analysis of a continuous oil pipeline against transverse permanent lateral spreadinginduced ground deformation to evaluate the sensitivity of the response to the width of the sliding mass and the characteristics of the pipe. The study was performed using the finite element code ANSYS. The structure was defined using 4-node shell elements, while soil-structure interaction is addressed through three-dimensional soil springs. Soil springs within a lateral spreading zone are assumed to have the same horizontal and vertical displacement, while the springs outside these zones are fixed. The elastic-plastic hardening model represents the behaviour of steel pipe, while an elastic-perfectly plastic law is adopted to define the soil. The lateral spreading was taken into account by applying a displacement field. In this study, lateral spreading widths (referred as $W$ ) of $10 \mathrm{~m}, 30 \mathrm{~m}$, and $50 \mathrm{~m}$ were considered. Liu and O'Rourke [51] found that this parameter has a great influence on the tensile strain of pipelines. A steel pipe of diameter $D=0.8 \mathrm{~m}$ and thickness $t=0.009 \mathrm{~m}$ was adopted in the analyses and, as a result, except for lateral spreading width corresponding to $10 \mathrm{~m}$, the peak tensile strain ended up being larger than the peak compressive strain, while the maximum pipe strain is reached for $W=30 \mathrm{~m}$. On the contrary, compressive strain reaches its maximum level for $W=10 \mathrm{~m}$, in contrast with the findings of Liu and O'Rourke [51]. This misprediction could be related to the beam element used by Liu and O'Rourke [51], which is not considered able to simulate 3D effects. Furthermore, for the case with lower lateral spreading width, peak strains reach the highest value with lower displacement than that required for the larger lateral spreading zones considered in the study. It is worth noting that for high $W$, the pipeline is observed to behave like a soft cable, while for lower widths, behaviour of continuous pipes is closer to a beam. Considering a parametric variation of pipe diameter $(0.4 \mathrm{~m}$, $0.6 \mathrm{~m}$ and $0.8 \mathrm{~m}$ diameters are considered), it was confirmed that a slower increase in maximum pipe strain is seen with increasing pipe diameter. Finally, incrementing the pipe thickness $(8 \mathrm{~mm}, 9 \mathrm{~mm}$ and $12 \mathrm{~mm}$ thicknesses are taken into account), it was observed that the pipe strain decreases. 
However, the correlation between the latter parameter and the maximum pipe strain appears weak.

Sahoo et al. [52] performed 3D numerical analyses considering both the cases of single- and double-embedded pipelines, in order to evaluate the effects of the pipeline spacing and burial depth on the pipe response subjected to horizontal load. The layout consists of the structure surrounded by gravelly soil, which works as a drainage system, and liquefiable sandy soil. The soil was meshed through 10node tetrahedron elements, while the pipeline was defined through plate elements using 8-nodesquadratic elements. The elastoplasticity of the soil was taken into account using the Mohr-Coulomb model while interface elements were located around the pipe, and normal and shear stiffness moduli, cohesion, internal friction angle, and a strength reduction factor were assigned to define them. As input motion, an accelerogram with a maximum amplitude of $0.247 \mathrm{~g}$ was selected and applied in the horizontal direction, orthogonally to the pipe. The analyses were performed through the finite element software MIDAS/GTS. It was found that, considering the layout of a single pipeline, the highest horizontal displacement is observed at a burial depth equal to the pipe diameter. A single pipe with $0.015 \mathrm{~m}$ thickness and $2.1 \mathrm{~m}$ diameter was considered with different burial depths of $1.05 \mathrm{~m}, 2.1 \mathrm{~m}$, and $4.2 \mathrm{~m}$ (i.e., equal to half a diameter, one diameter, and two times the diameter, respectively). The maximum displacement, observed in the centerline of the pipe, belongs to the range between less than $0.015 \mathrm{~m}$ and $0.018 \mathrm{~m}$. Considering the case of two pipelines, the impact of the spacing between the structures is investigated. The test layout consisted of a fixed burial depth corresponding to the outer diameter, with different spacings, namely, $1.05 \mathrm{~m}, 2.1 \mathrm{~m}$, and $4.2 \mathrm{~m}$. Higher displacements than the previous case are observed; the value of about $0.025 \mathrm{~m}$ is reached, with a less evident range of variability. However, the maximum value of displacement is obtained when the spacing is equal to half the pipe, while it is minimum when the spacing corresponds to the pipe diameter. Furthermore, as the spacing increases, there is a tendency to develop stress.

A strong effort to simulate the pipeline response under seismic loads, with a specific focus on the crossing of active faults, was taken within the GIPIPE research project [53]. In this framework, Sarvanis et al. [39] performed a wide experimental program of numerical analyses with a view to evaluate the parameters to be defined for a proper prediction of the soil-structure interaction when a pipeline is subjected to deformations generated by an earthquake. In the finite element context, 4-node reduced-integration shell elements were adopted to define the cylindrical pipeline, while 8-node reduced-integration brick elements were selected for the soil. Thus, the chosen approach imposes that the soil behaviour needs to be characterized through an appropriate constitutive model. For this reason, the model developed by Anastasopoulos et al. [54] was adopted. The latter represents a development of the classical Mohr-Coulomb theory. Its reliability was widely evaluated (see Sarvanis et al. [55]), mainly for the description of the softening behaviour of granular soils. The model requires a direct shear test to define both the plastic peak shear strain and the residual shear strain, where the latter is useful to define the dilatancy angle. The pipe obeys to a von Mises model. The finite element software ABAQUS was adopted in order to simulate the behaviour of a $1 \mathrm{~m}$ width of the soil-pipe system by applying a displacement-controlled pattern and allowing the pipe to move in the horizontal transverse direction, while any vertical displacement is forbidden. This was made to reproduce full-scale tests. Even if the model is not able to simulate all the physical liquefaction-induced phenomena or, more generally, large deformation effects of cohesionless soils, the results are in good accordance with physical tests and the interaction appears to be well reproduced. Once the model was calibrated, a parametric analysis was conducted to estimate the effect of the constitutive formulation on the response, by considering (1) the classical Mohr-Coulomb formulation with the peak friction angle, (2) the classical Mohr-Coulomb formulation with residual friction angle and null dilation angle, and (3) the modified abovementioned Mohr-Coulomb formulation. Results showed an overestimation of the laboratory test displacement if the peak friction angle is used, while similar results and better fitting of the physical tests are provided by the two latter conditions, indicating that a complex constitutive formulation can be avoided by using residual friction angle in the classical Mohr-Coulomb model. Moreover, a comparison with the prescriptions provided by the existing design guidelines seems to show good accordance. However, this does not mean that numerical accuracy is not required.

In the work proposed by Zou et al. [56], the uplift problem was analysed by using the finite element method. A coupled stress-flow finite element procedure, considering the u-p formulation of dynamic Biot's equations [57] and a hyperbolic stress-strain relationship, was exploited. Pore water pressure is taken into account through the model developed by Seed et al. [58]. The effectiveness of the approach is validated by simulating shaking table tests. In the finite element model, isoparametric quadrilateral elements with four solid nodes and four fluid nodes were deployed. The model was excited with a transient acceleration time history of PGA equal to $0.6 \mathrm{~g}$. It emerged that the excess pore water pressure close to the bottom of the pipe obtained with the FE analysis provided higher values than those encountered in the physical model; indeed, a value of about $2 \mathrm{kPa}$ was reached in the numerical results, while $0.5 \mathrm{kPa}$ was recorded during the experiments. The authors relate this issue to the gap forming between the pipe and soil that was not modelled in the analysis. Considering the sensor located close to the top of the pipe, the shaking table tests report a faster dissipation that is not simulated numerically and a value of $0.5 \mathrm{kPa}$ is attained until the end. This was probably related to the cracks developed at the sand surface generated by the pipeline uplift. Furthermore, qualitative accordance is observed comparing excess pore water pressure near to the springing depth of the pipe where $1 \mathrm{kPa}$ is recorded at the end of the excitation.

Ling et al. [59] developed a coupled stress-flow finite element procedure considering the dynamic Biot equations [60] with a view to analyse the embedded pipe uplift in liquefiable soil. Results were compared to centrifuge tests. 
The soil consisted of loose Nevada san,d and the response was examined in terms of excess pore water pressure, acceleration, earth pressure, and uplifting of the pipe. The simulations were performed using the finite element DIANA-SWANDYNE II code that can reproduce 2D boundary value problems. To account for the Biot dynamic equations, the $\mathrm{u}-\mathrm{p}$ formulation was adopted (where $u$ indicates the soilskeleton displacement and $p$ represents the pore pressure), without considering the relative acceleration of fluid with respect to the soil skeleton. The soil constitutive model is the Pastor-Zienkiewicz Mark-III ([61, 62]) that deploys the generalized plasticity theory. A set of calibrated parameters is available from Chan et al. [63] for Nevada sand. The constitutive model has shown a satisfying capability to simulate sand behaviour under cyclic loading conditions. Two different tests were performed to evaluate the response of the soil in the far-field condition and including the pipe. In the model, the laminar box of the centrifuge was defined through tied nodes, while 4-node quadrilateral elements were used for the pipe, which is a nonporous elastic material. A $0.6 \mathrm{~g}$ acceleration was adopted as input motion. The obtained results are in good agreement with the experimental recordings. However, considering in detail simulated and recorded acceleration time histories around the pipe, some discrepancies are observed in terms of amplitude. Referring to the simulated accelerograms, after a higher peak is attained, a decreasing trend in the acceleration response is observed until the peak-to-peak amplitude reaches a constant value after 2.5-3 s. The simulations at the springing depth of the pipe show a constant peak amplitude after $3 \mathrm{~s}$ in the range $0.1-0.2 \mathrm{~g}$. Slight differences are encountered by comparing these results with the recordings. On the contrary, strong differences are detected in the time interval $1.5-3 \mathrm{~s}$, where in the numerical simulation, a decreasing response is observed after a peak value of about $0.4 \mathrm{~g}$, while the recordings show maximum accelerations always in the range $0.1-0.2 \mathrm{~g}$. Referring to the crown depth of the pipe, differences are more pronounced for the whole time history: before $2.5 \mathrm{~s}$, maximum amplitudes of about $0.3-0.4 \mathrm{~g}$ were reached in the simulations, while the recordings show the maximum amplitude of $0.2 \mathrm{~g}$. Considering the response after $2.5 \mathrm{~s}$, maximum amplitudes of $0.2 \mathrm{~g}$ are recorded in both cases, but the experimental response seems to be translated from null acceleration value, suggesting a permanent displacement of the sensor. Furthermore, at the invert depth, the simulated response reaches constant peak amplitude between $0.2 \mathrm{~g}$ and $0.3 \mathrm{~g}$ after $2.5 \mathrm{~s}$, while higher peaks between $0.5 \mathrm{~g}$ and $0.6 \mathrm{~g}$ are reached earlier. Considering the recorded response, a peak amplitude of $0.3 \mathrm{~g}$ is observed but a nonzero mean value is encountered. For the excess pore water pressure generation, the simulations exhibited higher values than those obtained experimentally. Maximum values of $50 \mathrm{kPa}$ were attained numerically at the end of the shaking, while maximum values lower than $40 \mathrm{kPa}$ were recorded in the physical campaign. However, the trends for both accelerations and excess pore water pressures seem to be well captured. These slight differences can be related to the soil-structure interaction due to the separation between soil and pipe that is not considered in the numerical simulations. This issue could be the cause of the lower uplift, about $10 \mathrm{~cm}$, at the end of the shaking exhibited by the analysis compared to the displacement measured during the experimental test that amounts to about $10.5 \mathrm{~cm}$. Furthermore, it is worth noting that preliquefaction behaviour is well simulated, but difficulties grew up in simulating the postliquefaction phase, where the real trend of the response was not well caught from the simulations. However, the numerical predictions could better fit the experimental tests adopting a soil constitutive model that reproduces more reliably the sand cyclic behaviour, while a proper soil-pipe interface could be considered using interface elements that allow seepage.

Saeedzadeh and Hataf [64] adopt the finite element strategy within the software Plaxis to evaluate the effects of several parameters on the liquefaction-induced uplift of buried pipelines. The dilatancy angle, the relative density of soil, the burial depth, the diameter of the pipe, the groundwater table, and the thickness of the saturated soil were considered. A two-dimensional model was generated, and the hardening soil constitutive model developed by Schanz and Vermeer [65] was adopted. The latter includes soil dilatancy within the framework of the theory of plasticity and exploits a yield cap that closes the Duncan and Chang [66] hyperbolic model. The Nevada sand is considered, and the main parameters are obtained from the VELACS project [67]. The model calibration was performed by reproducing the tests conducted by Ling et al. [68]. Overall, the maximum amount of uplift is observed for a prescribed dilatancy angle in medium to dense sands, while in dense sands, the uplift tends to increase as the dilatancy angle decreases. The uplift increases as well when the relative density of soil decreases, and with a higher relative density, the burial depth behaves as an effective countermeasure to limit the phenomenon. Relative densities of $40 \%, 50 \%, 60 \%$, and $70 \%$ are taken into account and burial depths of $1.5 \mathrm{~m}$ and $3 \mathrm{~m}$ are analysed. For both the depths considered and a relative density of $40 \%$, the same ratio between uplift and burial depth of about 0.065 was reached. Instead, considering $70 \%$ relative density, the ratio is about 0.045 for $1.5 \mathrm{~m}$ burial depth, while it is slightly lower than 0.03 in the other case. The effect of burial depth alone highlights that moving from a ratio between depth and diameter of 0.5 to 1.5 , the uplift ratio varies from 0.14 to 0.03 . Moreover, the larger the pipe diameter, the higher the uplift. A closely linear relationship exists between the reduction in buoyancy and the drop of the water table. Furthermore, the negative effect of the water that increases both the reduction in the effective stress and the buoyancy force is observed. The increase in saturated soil thickness reduces the floatation because the distance from the excitation source lessens.

Chian et al. [69] analysed the problem of pipeline uplift induced by liquefaction using the Fast Lagrangian Analysis of Continua (FLAC) code, a widely adopted finite-difference program code in geotechnical field, which exploits Lagrangian calculation scheme and mixed-discretization zoning technique without matrices formed. Centrifuge tests were followed by numerical analyses with a view to assessing the variability of the pipeline response along with the depth and its sensitivity to the input motion. The numerical model 
was developed by adopting, for the soil, the bounding surface formulation proposed by Wang et al. [70], which consists of a fully coupled plasticity model for sands. The Hostun sand was considered for the surrounding soil and, consequently, the required input parameters for the constitutive model are $G_{0}$ (small-strain shear modulus), $h_{r}$ (shear modulus reduction with strain magnitude), and $k_{r}$ and $d$ (blow-count related parameters). The latter were obtained with a calibration procedure to achieve an analogous behaviour as in cyclic triaxial tests executed on the same sand (see, for instance, Bouferra et al. [71]). The soil-structure interaction was taken into account through a reduced friction angle. Moreover, the problem was assumed to be plane and the structure was considered rigid, while sinusoidal input motion is generated adopting upward propagating seismic waves. The experimental campaign highlights that excess pore water pressure, $\Delta u$, builds up contemporarily to the application of the excitation, but uplift takes place only during the motion, ceasing when no input is applied. A $0.2 \mathrm{~g}$ sinusoidal input motion was applied at the base. Considering a 1.1 ratio between burial depth and diameter of the pipeline, the uplift displacement reached the value of about $0.8 \mathrm{~m}$ in the experimental campaign and about $0.75 \mathrm{~m}$ in the numerical simulations after $27 \mathrm{~s}$ (i.e., at the end of the input motion), when $r_{u}$ is 0.9 . At this time, the displacement stops to increase. This evidence is also confirmed by assuming a ratio between burial depth and diameter equal to 1.5 . The value of $0.6 \mathrm{~m}$ of uplift displacement is reached after $27 \mathrm{~s}$ in both numerical simulations and centrifuge tests. In addition, considering a sinusoidal waveform of amplitude $0.1 \mathrm{~g}$ as input motion, a reduction of the uplift was observed. This underlines that the input seismogram decisively affects the phenomenon and, in particular, when a greater magnitude of input accelerations is applied, higher displacements are produced. When a total liquefaction stage takes place, the uplift reaches a constant rate and this can induce the conclusion that the soil-structure interaction leads to a limit in the overlying soil shear modulus reduction. Overall, satisfying accordance was observed in the comparison between numerical and experimental results, but the numerical simulations show that, in this case, the uplift starts earlier. Thus, shallower embedments result in lower resistance to the uplift due, probably, to the reduced extensions of failure planes and lower weight of the covering soil. Comparing the pore water pressure measurements near the pipe, the numerical results generally underestimate the mean observed response in correspondence of the invert and the springing of the structure, but they generally reproduce the same trends observed during the experimental campaign. The delayed uplift highlights that soil-structure interaction could be strongly influenced by the meshing scheme. Nonneglectable differences are observed at the crown, where the greater mean excess pore water pressure showed by the numerical results could be directly linked to a lower suppressed dilation due to liquefaction in the overlying soil. Finally, the typical deformation of soil around the structure is well replicated.

Sharafi and Parsafar [72] performed bidimensional analyses using the finite-difference software FLAC 2D to examine how some parameters of the soil-embedded pipeline system influence the uplift behaviour of the pipeline. The parameters considered in the study are soil dilatancy angle, relative density, friction angle, pipe diameter, and burial depth. Only half of the model is reproduced considering the symmetry of the problem and the homogeneity of the soil properties. To simulate liquefaction effects, the Finn model was adopted [73] for Nevada sand. The Nevada sand properties are obtained from the VELACS project [67]. Beam elements are selected to model the pipeline while the soil is modelled through square elements. Strong motion recordings are adopted as inputs (Naghan and Tabas earthquakes) as well as a $0.6 \mathrm{~g}$ amplitude sinusoid. The calibration procedure is based on the comparison with centrifuge test results from Ling et al. [68]. The validation procedure was useful to determine the interface strength reduction factor (namely $R_{\text {inter }}$ ) that takes into account the soil-structure interaction: a coefficient equal to 0.38 was obtained. Moreover, most of the results obtained from Saeedzadeh et al. [64] are confirmed. The sinusoidal motion was adopted to evaluate the soil dilatancy angle effect on the uplift with a layout consisting of a $1.5 \mathrm{~m}$ burial depth and a $3 \mathrm{~m}$ pipe diameter. It emerged that a peak in terms of uplift $(10.05 \mathrm{~cm})$ is reached for a dilatancy angle of $2.3^{\circ}$, while for higher angles, $3.7^{\circ}$ and in this case, the observed uplift decreases to $9.1 \mathrm{~cm}$. The same layout was considered to evaluate the influence of the relative density of the soil and Tabas Earthquake was adopted as input: an uplift of $6.85 \mathrm{~cm}$ was reached considering a relative density equal to $40 \%$, while referring to a relative density of $60 \%$, the final displacement lessens to $4.30 \mathrm{~cm}$. It is worth noting that for relative densities higher than $50 \%$, the rate of uplift decrease tends to 0 . Referring to the friction angle of the soil, uplift varies from $40 \mathrm{~cm}$ to $6 \mathrm{~cm}$ considering $15^{\circ}$ to $35^{\circ}$ friction angles, respectively. This underlines the strong effect of this parameter. Furthermore, considering the pipe diameter, the consequent uplift changes from $6.10 \mathrm{~cm}$ to $11.39 \mathrm{~cm}$ as the ratio between the diameter and the buried depth moves from 0.33 to 0.83 . Finally, it was observed that as the burial depth increases, the uplift decreases but the rate of the decrease evidently reduces when the burial depth passes the pipe diameter.

In their work, Marinatou et al. [74] found, differently from what is reported in codes and guidelines and from what was found in other research studies, that the soil overlying the pipe does not fully liquefy, thus forming a nonliquefied soil wedge that guarantees an appreciable shear strength above the pipe. 2D numerical analyses using the finitedifference code FLAC were performed. The soil constitutive behaviour is simulated through the NTUA-sand model $([75,76])$ which exploits the bounding surface theory. The centrifuge tests performed by Chian [69] were reproduced to validate the numerical model. Edges are modelled through tied-node boundaries that are not able to guarantee energy dissipation so that possible reflections can take place. However, the high-strain level is mobilized and the developed high hysteretic damping should absorb a large amount of the energy. Soil-aluminium pipe interface is simulated through contact elements with zero cohesion and adhesion 
angle equal to one half of the soil friction angle. A critical issue is represented by the constitutive model calibration. In fact, the selected constitutive model was calibrated with experimental results on Nevada sand, while Chian et al. [69] performed centrifuge tests on Hostun sand. To overcome this difficulty, a recalibration being unavailable, a higher relative density than that of the sand in the centrifuge tests was assumed in the model and trial-and-error simulations were performed to reproduce the liquefaction resistance developed by Bouferra et al. [71] with the NTUA-sand constitutive model. Observing the comparison between the results obtained in the analyses and physical tests performed by Chian et al. [69], it emerged that the excess pore water pressure at the crown reaches a lower peak value in terms of excess pore water pressure ratio than that attained at the springing and at the crown. This represents the proof that the soil above the pipe does not fully liquefy. This phenomenon could be related to the dilation and the dissipation effects at the free ground surface. In addition, a strong fluctuation of the excess pore pressure in each cycle (through dilation spikes) is observed in the numerical analysis. This finding contrasts with the experimental results, and it could be due to the high-frequency filtering in the centrifuge or due to the overprediction of dilation behaviour given by the constitutive model. However, once the model has been calibrated, different parameters are considered in the performed sensitivity analysis. They can be subdivided into three categories: geometry, soil properties, and seismic excitation. In detail, some of the abovementioned parameters were considered with reference to the following ranges: $0.6-1.2$ for the embedment ratio, $0.3 \mathrm{~m}$ to $1.5 \mathrm{~m}$ for the pipe diameter, $0.07-0.28$ for the cyclic resistance ratio (CRR), $30 \%$ to $75 \%$ for the relative densities, $10^{-6}$ to $10^{-3} \mathrm{~m} / \mathrm{s}$ for the permeability, and a factor of safety against liquefaction from 0.33 to 1.0 . It emerged that a model width of 15 pipe diameters is needed to eliminate undesired boundary effects. It is also evidenced that the increase in pipe diameter brings to an arising uplift displacement which reaches the value of about $3.5 \mathrm{~cm}$ for $1.5 \mathrm{~m}$ diameter; contextually, a higher displacement is observed in case of permeability reduction, while for soil with permeability lower than $10^{-4} \mathrm{~m} / \mathrm{s}$, uplift appears to be insensitive against this parameter. Furthermore, a greater relative density value lowers the uplift and this can be explained as a consequence of the higher dilation. A comparison is also made between the results obtained with the simplified methodology of Sasaki and Tamura [28] and the numerical predictions; nonneglectable mismatch outcomes for very loose soils $\left(D_{r}=30 \%\right)$; low-frequency motions (period higher than $T=0.7 \mathrm{~s}$, but this parameter is not properly taken into account in the simplified method); and high liquefaction risk $(F S=0.33)$, where the simplified method tends to overpredict the uplift displacement.

Xia and Zhang [77] performed finite element analysis using the ABAQUS code to validate their analytical model, which explicitly considers axial forces on pipe bending response by coupling both axial and flexural responses of the pipe. In the model, three-dimensional elements (defined as PIPE31) already implemented in the program code were used for the structure; a finer mesh was adopted to model the pipe and the soil adjacent to the liquefaction zone, while a coarser mesh is adopted to model the soil elsewhere. This choice is related to the high strain level that is expected in the liquefaction zone. The length of the pipe included in the liquefaction zone is assumed to be one-ninth of the total length of the model to avoid boundary effects. Soil-pipe interaction in the nonliquefaction area is defined using the PSI34 element provided by ABAQUS, to constrain the buried structure. An API Grade X70 steel pipeline was surrounded by soil with peak soil resistance equal to $126.9 \mathrm{kN} / \mathrm{m}$ and a consequent yield displacement of $0.18 \mathrm{~m}$. These parameters need to be assigned for the definition of the soil spring stiffness, which results to be $700 \mathrm{kN} / \mathrm{m}^{2}$. Three different cases are analysed considering different pipe thicknesses and lengths crossing liquefaction zones. In this process, a buried pipe with a $914 \mathrm{~mm}$ diameter and $13.1 \mathrm{~mm}$ thickness is used with a burial depth of $1.8 \mathrm{~m}$. The resulting uplift displacement varies from about $0.15 \mathrm{~m}$, obtained considering $30 \mathrm{~m}$ of liquefaction-area pipe crossing, to about $0.55 \mathrm{~m}$ resulted assuming $50 \mathrm{~m}$ of pipe length in the liquefied zone. A gap between the analytical response developed by the authors and the finite element model is observed. This can be related to the geometric nonlinearity and to the elastoplasticity considered in the finite element model only, while the analytical model considers exclusively the soil uplift resistance.

An overview of computational methods adopted to evaluate the stability of onshore pipelines in liquefiable soils was also provided by Castiglia et al. [78]. The mechanism of floating is briefly recalled; the simplified formulation for the safety factor in case of the uplift mechanism is commented with some numerical examples based on API Specifications, by comparing different cases of practical relevance, and some advanced approaches, usually adopted, that include numerical codes and the choice of adequate constitutive modelling are reported with recent trends in the geodisaster field numerical tools.

3.2. Limitations. In the case of liquefaction, soil stress-strain behaviour is very difficult to be modelled numerically. Moreover, the development of excess pore water pressure in undrained conditions is a crucial parameter to be simulated, the cause of the phenomenon being itself and extensive damage.

The replication of the in situ lateral spreading conditions through physical tests is extremely arduous. Consequently, the numerical models that aim at simulating this particular phenomenon cannot be easily developed because of the problems connected to the calibration procedure. Indeed, very often, the models to evaluate the response of pipelines crossing active faults are used in lateral spreading conditions as well. This can generate mispredictions linked, for example, to the unconsidered liquefaction of the surrounding soil.

Uplift problems appear easier to be modelled through physical tests, but the soil-pipe interaction in numerical simulations is generally difficult to be reproduced. As it was observed, there are always errors in the prediction of the 
response closer to the pipeline. This happens because the response is strongly affected by local mechanisms (e.g., local cracks in the soil overlying the pipeline due to the uplift) that are challenging to be simulated.

The modelling process is often very difficult to be attained because a reliable computational analysis needs a lot of parameters to be defined. Unfortunately, in the practice, there is a strong connection with the economic aspect, and for this reason, proper soil characterization is not always available.

Regarding the soil definition, a lot of constitutive models capable to simulate liquefaction are available in the literature, even though being developed and calibrated for a specific material. Furthermore, some mispredictions for high strain levels can be generated; for this reason, the postliquefaction behaviour of the soils is very difficult to be evaluated, even applying very advanced constitutive models.

A key role is also played by the meshing technique. Indeed, a proper meshing procedure of the analysed dominion has to ensure that a high displacement field is allowed, and this can affect the computational time. In addition, the seepage is not often considered in the analyses and this can lead to misleading evaluation of the model response. Finally, in the real observations, there is a coupling between horizontal and vertical displacements, while in the analysis, this condition is difficult to reproduce.

\section{Analytical Methods for Pipeline Performances}

The analytical methods presented here come from a geotechnical analysis of the phenomena without a focus on the stresses and strains imposed on the structure itself, which would need to account for structural characteristics as well and is out of the scope of this study. Moreover, among the analytical approaches available in the literature, only easily adopted simplified methods are mentioned in this paragraph, with relevance for practical applications. This means that additional research is available on the topic, but often the input data are unknown or of difficult determination, thus making the simplified procedure not worth using.

4.1. Findings. In the following, commonly used analytical approaches for the evaluation of vertical and horizontal forces acting on the pipe within liquefied soils are recalled, referring to the pipeline transverse cross section.

Regarding the uplift, the upward force exercised on the pipeline can be easily computed on the basis of a vertical equilibrium of forces acting on a transversal cross section (Figure 2), as commonly assumed in the research (e.g., $[27,32,33,68])$ and, consequently, in the standard of practice. The equilibrium of forces is given in equation (1). $W_{\text {pipe }}$ and $W_{\text {soil }}$ are, respectively, the weight of the pipe and the weight of the overlying soil column, $Q$ is the friction due to the potential vertical movement of the system, $U_{s}$ is the buoyant force due to hydrostatic pressure, $U_{D}$ is the uplift force due to the excess pore water pressure, $F$ is the seepage force transferred from the soil layer underlying the structure, $H$ is the burial depth, $D$ is the pipeline diameter, $\gamma$ ' is the soil effective unit weight, $\gamma_{f}$ is the unit weight of the fluidized material (equal to the soil saturated unit weight $\gamma_{\text {sat }}$ in case of complete liquefaction), $\gamma_{p}$ is the specific weight of the pipe, and $\Delta_{u}$ is the excess pore water pressure. Equation (1) is obtained under the assumptions of disregarding the seepage force of underlying layers (it is found to be less than $5 \%$ of $\left.U_{D}\right)$, considering the water level at the ground surface, and neglecting the frictional forces because of the soil strength loss with liquefaction occurrence:

$$
\frac{\pi D^{2}}{4} \gamma_{f}+\Delta_{u} \frac{\pi D}{2}=\frac{\pi D^{2}}{4} \gamma_{p}+\gamma^{\prime} D\left(H+\frac{D}{2}-\frac{\pi D}{8}\right) .
$$

Castiglia [34] made some additional consideration on the unit weight of the fluidized material in the simplified formulation of vertical equilibrium by confirming that, based on the results obtained through shaking table tests, it can be assumed equal to the saturated unit weight without substantial differences for the case of partial liquefaction. It is the procedure itself that is too conservative for the estimation of the safety factor in case of partial liquefaction of the soil deposit and not the unit weight itself, which has a very small influence on the results.

This conservative approach can be substituted by the procedure given by Huang et al. [79], which computes the uplift displacement considering the vertical forces acting on the pipe at each instant of time during the shaking and uses the principle of Newmark's sliding block to sum up the displacements in different intervals of time. In this way, it would be possible to include the frictional force in the equilibrium, to change the weight of the soil above the pipe while uplift proceeds and to account for the difference in excess pore water pressure development.

When structures are buried in saturated soils and subjected to horizontal earthquake vibrations, the lateral side of the pipe may be subjected to the combination of the dynamic water and dynamic effective soil pressure. Soil and water static pressures are applied on both sides of the embedded pipe and acting opposite to each other, thus making their contribution null and the dynamic pressure the only resultant on the buried structure. This cyclic component can be modelled by using the Westergaard [80] theory that was originally proposed for water reservoirs in dams. Wang et al. [81] studied the dynamic response of buried pipelines during liquefaction and, for a transversal section including a manhole, they computed the pressure on the rigid vertical wall by using Westergaard's equation with a correction factor to include the geometry of the wall. The horizontal pressure acting on the pipe $P$ can be computed by using the following equation:

$$
P=2\left(C \frac{7}{8} \gamma_{f} K_{h} \sqrt{\mathrm{HY}}\right) .
$$

In equation (2), $C$ is the correction factor, $K_{h}$ is the horizontal seismic coefficient, $\mathrm{H}$ is the height of the model, $\mathrm{Y}$ is the depth from the water surface, and $\gamma_{f}$ is the unit weight of the fluidized material (equal to the soil saturated unit weight $\gamma_{\text {sat }}$ in case of complete liquefaction). The application of the Westergaard theory to liquefied soil substitutes the unit weight of the water with the unit weight of liquefied soil. 


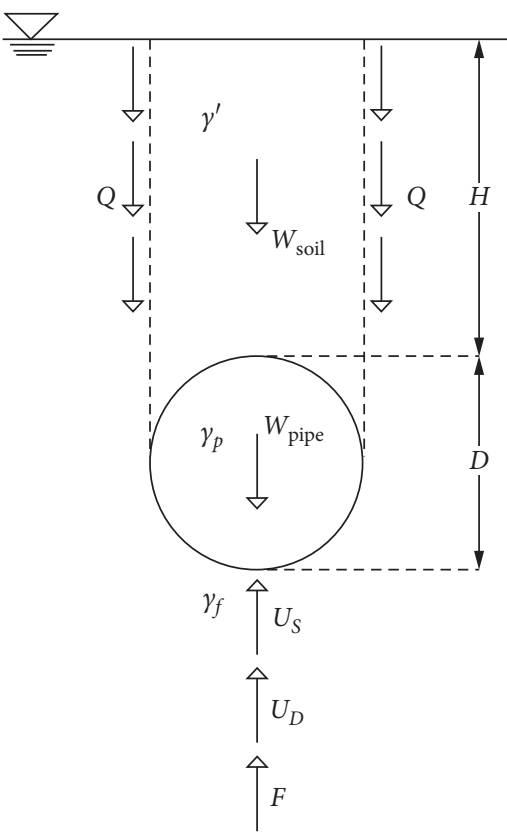

(a)

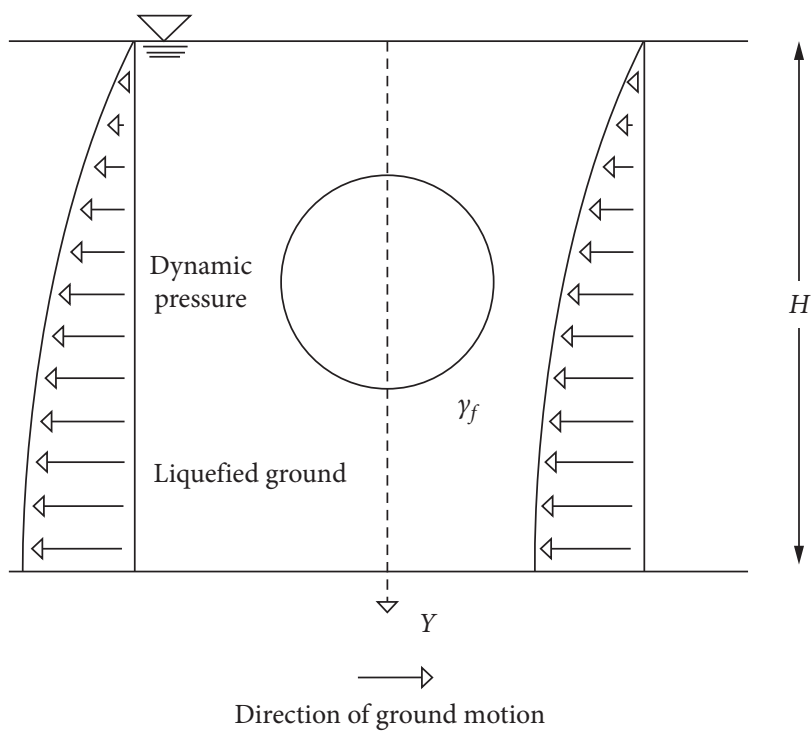

(b)

Figure 2: Simplified schemes of vertical equilibrium (a) and horizontal equilibrium (b).

If the problem is extended from the pipe's transversal cross section to the longitudinal development of the buried structure, additional elements need to be accounted for in the analytical solutions. The soil-pipe interaction can be modelled, in the simplest way, as a beam on elastic foundation, if it is within the range of elastic deformations. The interface between the pipe and soil is accounted for with elastic springs. The stiffness of the springs in liquefied soil can be computed using correction factors that can be applied to the stiffness of the spring for nonliquefied soils. Miyajima and Kitaura [82] stated that in liquefied soils, the soil spring constant is an increasing function of the effective stress and a decreasing function of excess pore water pressure ratio. O’Rourke et al. [83] suggested assuming both longitudinal and transversal stiffness around 3\% of that for nonliquefied soil. By following this approach, a soft-soil condition can be easily considered in the reduction of the spring coefficients, but the problem becomes not this much easy when a complete liquefied-state condition needs to be adopted. In this case, indeed, the soil acts like a viscous fluid and the relative velocity between the pipe and soil should be included.

When a large buoyancy displacement is expected, the maximum pipe displacement to prevent its breakage can be computed by referring to a beam of length equal to the length of the liquefied area, constrained at the extremities in correspondence to the nonliquefied soils. Hou et al. [84] proposed an equation based on the principle of conservation of energy for the computation of the maximum displacement $\delta_{\text {max }}$ :

$$
\delta_{\max }^{3}+\frac{16 I}{A} \delta_{\max }-\frac{16 P_{u} W_{s}^{4}}{A E \pi^{5}}=0 .
$$

In equation (3), $A$ is the cross section area, $I$ represents the moment of inertia, $W_{s}$ is the beam length (i.e., space between the restraints), $P_{u}$ is the maximum resistance in the horizontal transverse direction, and $E$ is the modulus of elasticity. For the exhaustive literature on analytical approaches including stresses and strains on pipelines, please refer to the work of O’Rourke and Liu [85].

4.2. Limitations. Even if the simplified methodologies represent a very useful tool to have a first assessment of the uplift or the permanent ground displacement related to lateral spreading, they are limited by the assumptions under which they are developed, their predictions becoming unreliable in case these conditions are not replicated. In some cases, caution is required not to oversimplify a complex problem through the use of correction factors or by neglecting some important contributions.

Even if the practical use of the abovementioned simplified methodologies is extremely advantageous for its timesaving, these methods often mispredict the displacements observed, carrying out physical tests, and for this reason, a more refined analysis is preferred. In this context, the computer analysis can decisively help the designer to test how the pipeline response is sensitive to the different parameters involved in the design process through, for example, parametric analysis.

\section{Conclusion and Future Assessments}

Protection from pipeline failure is one of the main concerns in the oil and gas industry due to potential serious implications in terms of economic loss and environmental concerns if the damages of such lifeline systems occur. 
Interesting data are provided by Dai et al. [86]. For pipeline network, risks control is another important issue (see, for instance, $\mathrm{Li}$ et al. [87]).

In seismic areas, the principal effects of liquefactioninduced ground deformations on pipelines are recalled here, with a focus on buoyancy and lateral spreading. Case histories reporting pipelines damage in the occurrence of liquefaction manifestations with main earthquakes are listed and briefly described, emphasizing the experience of failure modes and associated geological contexts and earthquake characteristics. Methods for experimental and numerical modelling of soil-pipe interaction in the occurrence of liquefaction, referring to uplift and lateral movement, are reviewed. The achieved outcomes of the research and the main limitations are described because these represent the build-up of the current state of knowledge. Case histories of real observations and experimental and numerical modelling are a very important source of understanding, and they all contribute to the improvement of seismic hazard effect preventions. This current state of knowledge is important, indeed, in view of future improvements of the sources of knowledge that can become more accurate, faster, and more effective by reducing the limitations; of the response to seismic hazard effects of pipeline networks with more effective prevention systems; and of the pipeline performances, also through the technologies adopted for pipeline equipment.

\section{Data Availability}

The data used to support the findings of this study are from the existing literature.

\section{Conflicts of Interest}

The authors declare that there are no conflicts of interest regarding the publication of this paper.

\section{References}

[1] M. Kitaura and M. Miyajima, "Quantitative evaluation of damage to buried pipelines induced by soil liquefaction," Proceedings of the 9th World Conference on Earthquake Engineering, pp. 11-16, Japan Association for Earthquake Disaster Prevention, Tokyo, Japan, 1988.

[2] T. L. Youd and S. N. Hoose, "Liquefaction during 1906 san Francisco earthquake," Journal of Geotechnical and Geoenvironmental Engineering, vol. 102, 1976.

[3] T. D. O'Rourke and M. S. Tawfik, "Effects of lateral spreading on buried pipelines during the 1971 san Fernando earthquake," ASME, vol. 77, pp. 124-132, 1983.

[4] J. Liang and S. Sun, "Site effects on seismic behavior of pipelines: a review," Journal of Pressure Vessel Technology, vol. 122, no. 4, pp. 469-475, 2000.

[5] Y. M. Lim, M. K. Kim, T. W. Kim, and J. W. Jang, "The behavior analysis of buried pipeline: considering longitudinal permanent ground deformation," in Proceedings of the Pipelines 2001, pp. 1-11, San Diego, CA, USA, July 2001.

[6] T. D. O'Rourke and P. A. Lane, Liquefaction Hazards and Their Effects on Buried Pipelines, National Center for Earthquake Engineering Research, Taipei, Taiwan, 1989.
[7] S. Yasuda, H. Nagase, S. Itafuji, H. Sawada, and K. Mine, “A study on the mechanism of the floatation of buried pipes due to liquefaction," WIT Transactions on The Built Environment, vol. 15, pp. 1-8, 1985.

[8] J. Koseki, O. Matsuo, and T. Sasaki, "Damage to sewer pipes during the 1993 kushiro-oki and the 1994 hokkaido-toho-oki earthquakes," Soils and Foundations, vol. 40, no. 1, pp. 1-13, 2000.

[9] S. Yasuda and H. Kiku, "Uplift of sewage manholes and pipes during the 2004 niigataken-chuetsu earthquake," Soils and Foundations, vol. 46, no. 6, pp. 885-894, 2006.

[10] S. Bhattacharya, M. Hyodo, K. Goda, T. Tazoh, and C. A. Taylor, "Liquefaction of soil in the tokyo Bay area from the 2011 Tohoku (Japan) earthquake," Soil Dynamics and Earthquake Engineering, vol. 31, no. 11, pp. 1618-1628, 2011.

[11] T. L. Holzer (Ed.), "The loma Prieta, California, earthquake of october 17, 1989: liquefaction," US department of the interior. US Geological Survey, Reston, VA, USA, 1998.

[12] T. D. O'Rourke, M. Grigoriu, and M. Khater, "Seismic response of buried pipeline; A state-of-the-art review," in Decade of Progress in Pressure Vessel Technology, C. Sundararajan, Ed., pp. 281-323, American Society of Mechanical Engineers, New York, NY, USA, 1985.

[13] T. D. O’Rourke and M. C. Palmer, "Earthquake performance of gas transmission pipelines," Earthquake Spectra, vol. 12, no. 3, pp. 493-527, 1996.

[14] T. D. O'Rourke and M. S. Tawfix, "Effect of lateral spreading on buried pipelines during the 1971 san Fernando earthquake," Earthquake Behavior and Safety of Oil Gas Storage Facilities, vol. 77, pp. 124-132, Buried Pipelines and Equipment, Boca Raton, FL, USA, 1983.

[15] M. Hamada, "Large ground displacement and buried pipe failure by soil liquefaction during 1983 nihonkai-chubu earthquake," in Proceedings of the 1985 ASME Pressure Vessel and Piping Conference, vol. 98, pp. 11-18, New Orleans, LA, USA, June 1985.

[16] M. Hamada, S. Yasuda, R. Isoyama, and K. Emoto, "Observation of permanent ground displacements induced by soil liquefaction," Doboku Gakkai Ronbunshu, vol. 376, pp. 211220, 1986.

[17] K. Wakamatsu, M. Hamada, T. Tazoh, N. Yoshida, and T. Ando, "Liquefaction and Landsliding from the July 16, 1990 Luzon, Philippine earthquake," in Proceedings of the 3rd USJapan Workshop on Earthquake Resistant Design of Lifeline Facilities and Countermeasures for Soil Liquefaction (NCEER91-0001), pp. 23-38, Kobe, Japan, December 1991.

[18] M. Hamada, K. Wakamatsu, T. Tazoh, and N. Yoshida, "Soil liquefaction and resulting damage to structures during the July 16, 1990, Philippines earthquake," The Japanese Geotechnical Society, vol. 39, no. 2, pp. 51-56, 1991.

[19] N. Nishio, "Mechanism of pipeline failures caused by soil liquefaction during the 1983 nihonkai-chubu earthquake," Doboku Gakkai Ronbunshu, vol. 556, pp. 53-63, 1997.

[20] J. P. Stewart, J. D. Bray, R. B. Seed, and N. Sitar, "Preliminary report on the principal geotechnical aspects of the january 17, 1994, Northridge earthquake," University of California, Berkeley, CA, USA, UCB/EERC-94/08, 1994.

[21] T. L. Holzer, M. J. Bennett, J. C. Tinsley, D. J. Ponti, and R. V. Sharp, "Causes of ground failure in alluvium during the Northridge, California, earthquake of January 17, 1994," in Japan-US Workshop on Earthquake Resistant Design of Lifeline Facilities and Countermeasures Against Soil Liquefaction, vol. 6, pp. 345-360, US National Center for Earthquake Engineering Research, Taipei, Taiwan, 1996. 
[22] R. M. Chung, The January 17, 1995 Hyogoken-Nanbu (Kobe) Earthquake. Performance of Structures, Lifelines, and Fire Protection Systems, National Institute of Standards and Technology (NIST Special Publication 901), Kobe City, Japan, 1996.

[23] M. R. Ghayamghamian, T. Tobita, S. Iai, S., and G. C. Kang, "Reconnaissance report of july 16, 2007 niigata-ken chuetsuoki, Japan, earthquake," Journal of Seismology and Earthquake Engineering, vol. 9, no. 1-2, pp. 73-84, 2007.

[24] M. Cubrinovski, H. Duncan, and B. A. Bradley, "Liquefaction impacts in residential areas in the 2010-2011 christchurch earthquakes," Tohoku Special Conference Paper 183, University of Canterbury, Christchurch, New Zealand, 2012.

[25] M. Cubrinovski, M. Hughes, B. A. Bradley et al., "Performance of horizontal infrastructure in christchurch city through the 2010-2011 canterbury earthquake sequence," Civil and Natural Resource Engineering, The University of Canterbury, Christchurch, New Zealand, 2014.

[26] GEER-Geoengineering Extreme Events Reconnaissance Association, Geoengineering Reconnaissance of the 2010 Maule, Chile Earthquake, Report of the NSF Sponsored GEER Association Team, Maule, Chile, 2010.

[27] J. Koseki, O. Matsuo, and Y. Koga, "Uplift behavior of underground structures caused by liquefaction of surrounding soil during earthquake," Soils and Foundations, vol. 37, no. 1, pp. 97-108, 1997.

[28] T. Sasaki and K. Tamura, "Prediction of liquefaction-induced uplift displacement of underground structures," in Proceedings of 36th Joint Meeting U.S.-Japan Panel on Wind and Seismic Effects, pp. 191-198, Gaithersburg, MD, USA, 2004.

[29] M. Stringer and S. G. P. Madabhushi, "Modelling of liquefaction around tunnels," in Proceeding of 4th International Conference on Earthquake Geotechnical Engineering, Thessaloniki, Greece, June 2007.

[30] K. Ichii, N. Seto, and H. Kidera, "Characteristics of uplifting velocity of a buried pipe in liquefied ground," Geotechnical Earthquake Engineering and Soil Dynamics, vol. 4, pp. 1-10, 2008.

[31] S. C. Chian and S. P. G. Madabhushi, "Effect of buried depth and diameter on uplift of underground structures in liquefied soils," Soil Dynamics and Earthquake Engineering, vol. 41, pp. 181-190, 2012a.

[32] S. C. Chian and S. P. G. Madabhushi, "Effect of soil conditions on uplift of underground structures in liquefied soil," Journal of Earthquake and Tsunami, vol. 06, no. 04, p. 1250020, $2012 \mathrm{~b}$.

[33] S. C. Chian, J. Wang, S. K. Haigh, and S. P. G. Madabhushi, "Soil deformation during monotonic and seismic pipe uplift in liquefiable soil," Journal of Pipeline Engineering, vol. 14, no. 1, pp. 33-41, 2015.

[34] M. Castiglia, The experimental study of buried onshore pipelines seismic-liquefaction induced vertical displacement in shaking table tests and its remedial measures, Ph.D. thesis, The University of Molise, Campobasso, Italy, 2019.

[35] C. H. Trautmann and T. D. O'Rourke, "Lateral force-displacement response of buried pipe," Journal of Geotechnical Engineering, vol. 111, no. 9, pp. 1077-1092, 1985.

[36] I. Towhata, W. Vargas-Monge, R. P. Orense, and M. Yao, "Shaking table tests on subgrade reaction of pipe embedded in sandy liquefied subsoil," Soil Dynamics and Earthquake Engineering, vol. 18, no. 5, pp. 347-361, 1999.

[37] S. A. Ashford and T. Juirnarongrit, "Response of single piles and pipelines in liquefaction-induced lateral spreads using controlled blasting," Earthquake Engineering and Engineering Vibration, vol. 1, no. 2, pp. 181-193, 2002.
[38] K. Shimamura, Y. Fujita, S. Kojima, Y. Taji, and M. Hamada, "Transverse horizontal load on buried pipes due to liquefaction-induced permanent ground displacement," Soils and Foundations, vol. 43, no. 1, pp. 59-73, 2003.

[39] G. C. Sarvanis, S. A. Karamanos, P. Vazouras, E. Mecozzi, A. Lucci, and P. Dakoulas, "Permanent earthquake-induced actions in buried pipelines: numerical modeling and experimental verification," Earthquake Engineering \& Structural Dynamics, vol. 47, no. 4, pp. 966-987, 2017.

[40] J. Zhang, H. Zhang, L. Zhang, and Z. Liang, "Buckling response analysis of buried steel pipe under multiple explosive loadings," Journal of Pipeline Systems Engineering and Practice, vol. 11, no. 2, 2020.

[41] I. Towhata, Geotechnical Earthquake Engineering, Springer Science \& Business Media, Berlin, Germany, 2008.

[42] T. K. Datta, "Seismic response of buried pipelines: a state-ofthe-art review," Nuclear Engineering and Design, vol. 192, no. 2-3, pp. 271-284, 1999.

[43] D. Chang, T. Travasarou, and J. M. Chacko, "Numerical evaluation of liquefaction-induced uplift for an immersed tunnel," Proceedings of the 14th World Conference on Earthquake Engineering, Seismological Press, Beijing, China, October 2008.

[44] H. Zhuang, Z. Hu, X. Wang, and G. Chen, "Seismic responses of a large underground structure in liquefied soils by FEM numerical modelling," Bulletin of Earthquake Engineering, vol. 13, no. 12, pp. 3645-3668, 2015.

[45] H. Liu and E. Song, "Seismic response of large underground structures in liquefiable soils subjected to horizontal and vertical earthquake excitations," Computers and Geotechnics, vol. 32, no. 4, pp. 223-244, 2005.

[46] G. Kouretzis and G. Bouckovalas, "Analysis of buried oil and gas pipelines crossing active faults: revisiting pipe-ground interaction," in Proceedings of the 7th International Conference on Geotechnical Earthquake Engineering, Beijing, China, October 2019.

[47] D. K. Karamitros, G. D. Bouckovalas, and G. P. Kouretzis, "Stress analysis of buried steel pipelines at strike-slip fault crossings," Soil Dynamics and Earthquake Engineering, vol. 27, no. 3, pp. 200-211, 2007.

[48] D. K. Karamitros, G. D. Bouckovalas, G. P. Kouretzis et al., "An analytical method for strength verification of buried steel pipelines at normal fault crossings," Soil Dynamics and Earthquake Engineering, vol. 31, no. 11, pp. 1452-1464, 2011.

[49] S. Takada, T. Suzuki, T. Koike et al., "Seismic design of buried pipelines for liquefaction-induced large ground displacement," Adv. Earthquake Eng.vol. 9, pp. 619-628, 2001.

[50] G. B. Shao, "Numerical analysis for oil pipeline subjected to earthquake liquefaction-induced lateral spreading," Advanced Materials Research, vol. 243-249, pp. 3804-3807, 2011.

[51] X. Liu and M. J. O'Rourke, "Behaviour of continuous pipeline subject to transverse PGD," Earthquake Engineering and Structural Dynamics, vol. 26, no. 10, p. 989, 1999.

[52] S. Sahoo, B. Manna, and K. G. Sharma, "Seismic behaviour of buried pipelines: 3D finite element approach," Journal of Earthquakes, vol. 2014, Article ID 818923, 9 pages, 2014.

[53] P. Vazouras, G. C. Sarvanis, S. A. Karamanos et al., "Safety of buried steel pipelines under ground-induced deformations," Final Report, GIPIPE Project, RFSR-CT-2011-00027, Research Fund for Steel and Coal (RFCS), Brussels, Belgium, 2015, https://publications.europa.eu/en/web/general-publications/ publications.

[54] I. Anastasopoulos, G. Gazetas, M. F. Bransby, M. C. R. Davies, and A. El Nahas, "Fault Rupture propagation through sand: 
finite-element analysis and validation through centrifuge experiments," Journal of Geotechnical and Geoenvironmental Engineering, vol. 133, no. 8, pp. 943-958, 2007.

[55] G. C. Sarvanis, J. Ferino, S. A. Karamanos et al., Soil-pipe Interaction Models for Simulating the Mechanical Response of Buried Steel Pipelines Crossing Active Faults, International Society of Offshore and Polar Engineers, Rhodes, Greece, 2016.

[56] D. Zou, X. Kong, and B. Xu B, "Numerical simulation of seimic behavior of pipeline in liquefiable soil," in Soil StressStrain Behavior: Measurement, Modeling and Analysis, H. I. Ling, L. Callisto, D. Leshchinsky, and J. Koseki, Eds., Vol. 146, Springer, Dordrecht, Netherlands, 2007.

[57] O. C. Zienkiewicz, "Basic formulation of static and dynamic behavior of soil and other porous media," in Numerical Methods in Geomechanics, J. B. Martin, Ed., Springer, Berlin, Germany, 1982.

[58] H. B. Seed, P. P. Martin, and J. Lysmer, "Pore-water pressure changes during soil liquefaction," International Journal of Rock Mechanics and Mining Sciences \& Geomechanics Abstracts, vol. 13, no. 7, pp. 323-346, 1976.

[59] H. I. Ling, L. Sun, H. Liu et al., "Finite element analysis of pipe buried in saturated soil deposit subjected to earthquake loading," Journal of Earthquake and Tsunami, vol. 2, no. 1, 2008.

[60] M. A. Biot, "Theory of propagation of elastic waves in a fluidsaturated porous solid. I. Low-Frequency range," The Journal of the Acoustical Society of America, vol. 28, no. 2, pp. 168-178, 1956.

[61] M. Pastor, O. C. Zienkiewicz, and A. H. C. Chan, "Generalized plasticity model for three dimensional sand behaviour," Constitutive Equations for Granular Non-cohesive Soils, pp. 535-550, CRC Press, Boca Raton, FL, USA, 1988.

[62] M. Pastor, O. C. Zienkiewicz, and A. H. C. Chan, "Generalized plasticity and the modelling of soil behaviour," International Journal for Numerical and Analytical Methods in Geomechanics, vol. 14, no. 3, pp. 151-190, 1990.

[63] A. H. C. Chan, O. O. Famyiesin, and D. M. Wood, "Numerical prediction for model no. 1," Verification of Numerical Proccedures for the Analysis of Soil Liquefaction Problems, pp. 87-708, Balkema, Delft, Nederlands, 1994.

[64] R. Saeedzadeh and N. Hataf, "Uplift response of buried pipelines in saturated sand deposit under earthquake loading," Soil Dynamics and Earthquake Engineering, vol. 31, no. 10 , pp. 1378-1384, 2011.

[65] T. Schanz and P. A. Vermeer, "Special issue on pre-failure deformation behavior of geomaterials," Geotechnique, vol. 48, pp. 383-387, 1998.

[66] J. M. Duncan and C. Y. Chang, "Nonlinear analysis of stress and strain in soil," ASCE Journal of the Soil Mechanics and Foundations Division, vol. 96, pp. 1629-1653, 1970.

[67] K. Arulmoli, K. K. Muraleetharan, M. M. Hossain et al., "VELACS: verification of liquefaction analyses by centrifuge studies-laboratory testing program," Soil Data Report, University of Southern California, Los Angeles, CA, USA, 1992, http://geoinfo.usc.edu/gees/velacs/S.

[68] H. I. Ling, Y. Mohri, T. Kawabata, H. Liu, C. Burke, and L. Sun, "Centrifugal modeling of seismic behavior of largediameter pipe in liquefiable soil," Journal of Geotechnical and Geoenvironmental Engineering, vol. 129, no. 12, pp. 10921101, 2003.

[69] S. C. Chian, K. Tokimatsu, and S. P. G. Madabhushi, "Soil liquefaction-induced uplift of underground structures: physical and numerical modeling," Journal of Geotechnical and Geoenvironmental Engineering, ASCE, vol. 140, Article ID 04014057, 2014.
[70] Z.-L. Wang, Y. F. Dafalias, and C.-K. Shen, "Bounding surface hypoplasticity model for sand," Journal of Engineering Mechanicsics, 10.1061/, vol. 116, no. 5, pp. 983-1001, 1990.

[71] R. Bouferra, N. Benseddiq, and I. Shahrour, "Saturation and preloading effects on the cyclic behavior of sand," International Journal of Geomechanics, vol. 7, no. 5, pp. 396-401, 2007.

[72] H. Sharafi and P. Parsafar, "Seismic simulation of liquefaction-induced uplift behavior of buried pipelines in shallow ground," Arabian Journal of Geosciences, vol. 9, no. 3, p. 215, 2016.

[73] G. Martin, W. Finn, and H. Seed, "Fundamentals of liquefaction under cyclic loading," Journal of the Geotechnical Engineering Division, ASCE, vol. 101, 1975.

[74] S. M. Marinatou, V. A. Zontanou, G. D. Bouckovalas et al., "Liquefaction induced uplift of pipelines: numerical modeling and parametric analyses," in Proceedings of the 3rd International Conference of Performance-Based Design in Earthquake Geotechnical Engineering, M. Taiebat, D. Wijewickreme, A. Athanasopoulos-Zekkos, and R. W. Boulanger, Eds., ,Vancouver, BC, USA, July 2017.

[75] K. I. Andrianopoulos, A. G. Papadimitriou, and G. D. Bouckovalas, "Bounding surface plasticity model for the seismic liquefaction analysis of geostructures," Soil Dynamics and Earthquake Engineering, vol. 30, no. 10, pp. 895-911, 2010.

[76] A. G. Papadimitriou and G. D. Bouckovalas, "Plasticity model for sand under small and large cyclic strains: a multiaxial formulation," Soil Dynamics and Earthquake Engineering, vol. 22, no. 3, pp. 191-204, 2002.

[77] M. Xia and H. Zhang, "Stress and deformation analysis of buried gas pipelines subjected to buoyancy in liquefaction zones," Energies, vol. 11, no. 9, p. 2334, 2018.

[78] M. Castiglia, F. Santucci de Magistris, and A. Napolitano, "Stability of onshore pipelines in liquefied soils: overview of computational methods," Geomechanics and Engineering, vol. 14, no. 4, pp. 355-366, 2018.

[79] B. Huang, J. Liu, P. Lin, and D. Ling, "Uplifting behavior of shallow buried pipe in liquefiable soil by dynamic centrifuge test," The Scientific World Journal, vol. 2014, pp. 1-15, Article ID 838546, 2014.

[80] H. M. Westergaard, Water Pressure on Dams during Earthquakes, pp. 418-433, Transaction of ASCE, Reston, VA, USA, 1931.

[81] L. R. L. Wang, J. S. Shim, I. Ishibashi, and Y. Wang, "Dynamic responses of buried pipelines during a liquefaction process," Soil Dynamics and Earthquake Engineering, vol. 9, no. 1, pp. 44-50, 1990.

[82] M. Miyajima and M. Kitaura, "Effects of liquefaction-induced ground movement on pipeline," in Proceedings of the Second US-Japan Workshop on Liquefaction, Large Ground Deformation and Their Effects on Lifelines, pp. 386-400, Ithaca, NY, USA, September 1989.

[83] M. J. O'Rourke and X. Liu, Failure Criterion for Buried Pipe Subject to Longitudinal Pgd: Benchmark Case History, US National Center for Earthquake Engineering Research (NCEER), Buffalo, NY, USA, 1994.

[84] A. Hou, J. Cai, and X. Liu, "Response calculation of oil pipeline subjected to permanent ground movement induced by soil liquefaction," in Proceedings of the China-Japan Symposium on Lifeline Earthquake Engineering, Beijing, China, June 1990.

[85] M. J. O'Rourke and X. Liu, Response of Buried Pipelines Subject to Earthquake Effects, University at Buffalo, Buffalo, NY, USA, 1999. 
[86] L. Dai, D. Wang, T. Wang et al., "Analysis and comparison of long-distance pipeline failures," Journal of Petroleum Engineering, vol. 2017, Article ID 3174636, 7 pages, 2017.

[87] M. Li, L. Liu, Y. Li et al., "Evaluating the risk of natural gas pipeline operation management in intuitionistic fuzzy linguistic environments," Mathematical Problems in Engineering, vol. 2018, Article ID 3960496, 13 pages, 2018. 